\title{
SYMBOLIC POWERS AND FREE RESOLUTIONS OF GENERALIZED STAR CONFIGURATIONS OF HYPERSURFACES
}

\author{
KUEI-NUAN LIN AND YI-HUANG SHEN
}

\begin{abstract}
As a generalization of the ideals of star configurations of hypersurfaces, we consider the $a$-fold product ideal $I_{a}\left(f_{1}^{m_{1}} \ldots f_{s}^{m_{s}}\right)$ when $f_{1}, \ldots, f_{s}$ is a sequence of generic forms and $1 \leq a \leq m_{1}+\cdots+m_{s}$. Firstly, we show that this ideal has complete intersection quotients when these forms are of the same degree and essentially linear. Then we study its symbolic powers while focusing on the uniform case with $m_{1}=\cdots=m_{s}$. For large $a$, we describe its resurgence and symbolic defect. And for general $a$, we also investigate the corresponding invariants for meeting-at-the-minimal-components version of symbolic powers.
\end{abstract}

\section{INTRODUCTION}

Let $\mathbb{K}$ be an infinite field. Partly due to the rich combinatorial structure and the ability of exhibiting extremal numerical behavior, star configurations of points in $\mathbb{P}_{\mathbb{K}}^{n}$ have attracted strong research interest. As a generalization, the next step is to consider certain union of complete intersection subschemes obtained by intersecting some hypersurfaces in $\mathbb{P}_{\mathbb{K}}^{n}$.

To be accurate, let $R=\mathbb{K}\left[x_{0}, \ldots, x_{n}\right]$ be a standard graded polynomial ring over $\mathbb{K}$ and denote its graded maximal ideal by $\mathfrak{m}$. Let $\mathcal{F}=\left\{f_{1}, \ldots, f_{s}\right\}$ be a set of forms in $R$ with $s \geq n+1$ and consider the hypersurfaces $\mathcal{H}=\left\{H_{1}, \ldots, H_{s}\right\}$ defined by them in $\mathbb{P}_{\mathbb{K}}^{n}$. Suppose that these forms are $c$-generic in the sense that any subset of size at most $c+1$ will form a regular sequence. Then, we will obtain a star configuration of hypersurfaces of codimension $c$ :

$$
V_{c}\left(\mathcal{H}, \mathbb{P}_{\mathbb{K}}^{n}\right):=\bigcup_{1 \leq i_{1}<\cdots<i_{c} \leq s}\left(H_{i_{1}} \cap \cdots \cap H_{i_{c}}\right) .
$$

It has been of great interest to study various algebraic, geometric and combinatorial properties of star configurations; see for instance [3], [12], [13], 18] and the references therein. The object we are mostly interested here is the defining ideal

$$
I_{c, \mathcal{F}}:=\bigcap_{1 \leq i_{1}<\cdots<i_{c} \leq s}\left\langle f_{i_{1}}, \ldots, f_{i_{c}}\right\rangle .
$$

It was observed by Geramita et al. in [13] that the study of the minimal graded free resolutions of $I_{c, \mathcal{F}}$ and its symbolic powers can be reduced to the linear monomial case, i.e., when $s=n+1$ and $\mathcal{F}=\left\{x_{0}, x_{1}, \ldots, x_{n}\right\}$. Consequently, these symbolic power ideals will have linear-like resolutions.

This idea was later made more precise by Mantero in [18 by the notion of Koszul stranded Betti table; see Definition 1.2 below. Actually he showed that these ideals have complete intersection quotients. Independently, in the linear monomial case, Biermann et al. 33 showed that these symbolic power ideals are symmetry strongly shifted and consequently have linear quotients.

2010 Mathematics Subject Classification. 13A15, 13A50, 13D02, 14N20, 52C35.

Keyword: Betti numbers, fold products, linear quotients, star configuration, symbolic power . 
We want to remark at this moment that the ideal $I_{c, \mathcal{F}}$ above is the specialization of the Stanley-Reisner ideal of a matroid complex. In particular, it is Cohen-Macaulay and consequently a basic double $G$-linkage technique can be applied. Notice that this is crucial in the discussions of [12] and [13], since it allows one to describe minimal generating sets of the ideal $I_{c, \mathcal{F}}$ and its symbolic powers. And this is of course the starting point of the description of minimal free resolutions.

The defining ideal $I_{c, \mathcal{F}}$ in (11) can also be studied from a different point of view. Fix positive integers $a, m_{1}, \ldots, m_{s}$ such that $a \leq m_{1}+\cdots+m_{s}$. Now, consider the ideal generated by the $a$-fold products of the forms $f_{1}, \ldots, f_{s}$ with the multiplicities $m_{1}, \ldots, m_{s}$ respectively:

$$
I_{a}\left(f_{1}^{m_{1}} \cdots f_{s}^{m_{s}}\right):=\left\langle f_{1}^{n_{1}} \cdots f_{s}^{n_{s}}: 0 \leq n_{i} \leq m_{i} \text { for each } i \text { such that } \sum_{i} n_{i}=a\right\rangle .
$$

The ideal defined above is known as the a-fold product ideal of the corresponding configuration

$$
\underbrace{f_{1}, \ldots, f_{1}}_{m_{1}}, \underbrace{f_{2}, \ldots, f_{2}}_{m_{2}}, \ldots, \underbrace{f_{s}, \ldots, f_{s}}_{m_{s}} .
$$

It follows from [13, Theorem 3.3 and Example 3.4] that $I_{c, \mathcal{F}}$ coincides with $I_{s-c+1}\left(f_{1} f_{2} \cdots f_{s}\right)$ for $m_{1}=\cdots=m_{s}=1$.

The $a$-fold product ideal of linear forms was originally introduced as a nice tool for determining the minimum distance of linear codes in the coding theory. They also emerge naturally when dealing with higher order Orlik-Terao algebra of hyperplane arrangements. Intricate algebraic and combinatorial properties of $I_{a}\left(f_{1}^{m_{1}} \cdots f_{s}^{m_{s}}\right)$ have also attracted the attention of many researchers; see for instance [1], [11, [21], 22], [23] and the references therein.

We will pay a closer attention to the ideal $I_{a}\left(f_{1}^{m_{1}} \cdots f_{s}^{m_{s}}\right)$ within this note. The first aim is to study its Betti table. For this purpose, throughout this note, we will always assume the following assumption.

Setting 1.1. Let $\mathcal{F}=\left\{f_{1}, \ldots, f_{s}\right\}$ be a set of forms in $R=\mathbb{K}\left[x_{0}, \ldots, x_{n}\right]$ and suppose that these forms are generic, i.e., they are $n$-generic. Furthermore, fix some positive integers $a, m_{1}, \ldots, m_{s}$ with $a \leq m_{1}+\cdots+m_{s}$.

Recall that in [18, Definition 6.5] Mantero introduced the following notion.

Definition 1.2. Let $1 \leq n_{1}<\cdots<n_{r}$ be integers and $I$ a homogeneous ideal in $R$ generated in degrees $n_{1}, \ldots, n_{r}$. We say that $I$ has a Koszul stranded Betti table if and only if there exists a positive integer $d$ such that the graded Betti number

$$
\beta_{i, j}(R / I) \neq 0 \quad \text { only if } j \in\left\{n_{h}+d(i-1): h=1, \ldots, r\right\}
$$

for $i \geq 1$.

Notice that if $r=d=1$ in the above definition, then the ideal $I$ has a linear resolution. Meanwhile, under the Setting 1.1, if all the forms in $\mathcal{F}$ are linear, then it is already known by Tohăneanu and Xie [24, Theorem 2.3] that the ideal $I_{a}\left(f_{1}^{m_{1}} \cdots f_{s}^{m_{s}}\right)$ has a linear resolution. Inspired by this achievement, we are interested in the following question.

Question 1.3. Under the Setting 1.1, is it true that the ideal $I_{a}\left(f_{1}^{m_{1}} \cdots f_{s}^{m_{s}}\right)$ has a Koszul stranded Betti table? 
Meanwhile, inspired by the recent work of Tohăneanu and his coauthors, we are also interested in the following questions.

Question 1.4. Under the Setting 1.1, is it true that

$$
I_{a+1}\left(f_{1}^{m_{1}} \cdots f_{s-1}^{m_{s-1}} f_{s}^{m_{s}+1}\right): f_{s}=I_{a}\left(f_{1}^{m_{1}} \cdots f_{s-1}^{m_{s-1}} f_{s}^{m_{s}}\right)
$$

for all $a \leq m_{1}+\cdots+m_{s}$ ?

Question 1.5. Under the Setting 1.1, is it true that

$$
I_{a}\left(f_{1}^{m_{1}} \cdots f_{s}^{m_{s}}\right)=\bigcap_{c=1}^{s}\left(\bigcap_{1 \leq i_{1}<\cdots<i_{c} \leq s}\left\langle f_{i_{1}}, \ldots, f_{i_{c}}\right\rangle^{\mu_{a}\left(i_{1}, \ldots, i_{c}\right)}\right),
$$

for

$$
\mu_{a}\left(i_{1}, \ldots, i_{c}\right):=\max \left\{0, a-\sum_{j \in[s] \backslash\left\{i_{1}, \ldots, i_{c}\right\}} m_{j}\right\} ?
$$

Here, any ideal with non-positive power is replaced by the ring $R$. And as usual, $[s]$ is the set $\{1,2, \ldots, s\}$.

Regarding the Question 1.4, when all the forms of $\mathcal{F}$ are linear, Anzis, Garrousian and Tohăneanu showed in [1, Proposition 3.5] that the $a$-fold product ideal having a linear resolution is closely related to the colon ideal having the expected form as in (2)), even without the generic assumption. If the linear forms in $\mathcal{F}$ are generic, then Tohăneanu and Xie provided a positive answer to the Question 1.4 in the proof of 24, Theorem 2.3], as a byproduct of establishing the linear resolution property of the $a$-fold product ideal. For their proof, a positive answer to the Question 1.5] is vital; cf. [24, Corollary 2.4]. And they used this to confirm a conjecture of Geramita, Harbourne and Migliore ([12, Conjecture 4.1]) regarding a primary-decomposition type formula for powers of ideals of star configuration of hyperplanes.

Indeed, Geramita, Harbourne and Migliore themselves proved their own conjecture up to saturation in [12, Corollary 4.9]. Using a similar technique, we will show in Theorem 2.4 that a similar phenomenon also happens for generic configurations of hypersurfaces. It is worth pointing out at this stage that the ideal $I_{a}\left(f_{1}^{m_{1}} \cdots f_{s}^{m_{s}}\right)$ is not unmixed in general (even when $m_{1}=\cdots=m_{s}$ ), let alone Cohen-Macaulay.

Notice that with regard to general ideals lacking mutigrading structure, even for monomial ideals in the generic forms in $\mathcal{F}$, weird phenomena emerge for the descriptions of associated primes, containment problems and the colon operations; see for example [18, Remark 3.2]. Therefore, one won't be surprised to see computations by the software Macaulay2 [14] showing that the three questions raised above have negative answers in general. Therefore, we are obliged to impose a technical condition as being strongly generic. This new term means that in addition to $\mathcal{F}$ being generic and the forms there sharing a common degree, the forms actually come from a common $\mathbb{K}$-linear space of $\mathbb{K}$-dimension $\operatorname{dim}(R)=n+1$. Although this assumption seems artificial, it is still natural in the sense that generic set of linear forms are strongly generic. Notice that the latter is the condition needed when we are dealing with star configurations of hyperplanes.

To some extent, the strongly generic terminology simply means being generic and essentially linear. And when this strong condition is satisfied, we show with ease in Proposition 3.2 that all the three questions above have positive answers. 
As the first main contribution of this note, we indeed prove in Theorem 3.7 that the $a$-fold product ideal $I=I_{a}\left(f_{1}^{m_{1}} \cdots f_{s}^{m_{s}}\right)$ of generic forms will have complete intersection quotients under the strongly generic condition. This will particularly imply that the $a-$ fold product ideal of generic linear forms has a linear resolution, recovering a key result of Tohăneanu and Xie in [24]. Our approach is inspired by the viewpoint of Geramita, Harbourne, Migliore and Nagel in [13] and the recent work of Mantero in [18]. Since we don't have to dwell on the primary-decomposition type problems, this approach is more straightforward, and hence shorter when compared with the work in [24].

The argument for the complete intersection quotients actually bears more fruit than one generally expects at the first glance. It allows us to compare its Betti table with that of the prototype monomial ideal, i.e., when the forms $f_{1}, \ldots, f_{s}$ are actually ring variables. In particular, an upper bound of the projective dimension of the ideal $I_{a}\left(f_{1}^{m_{1}} \cdots f_{s}^{m_{s}}\right)$ can be easily described; see our Corollary 3.11. To obtain such a comparison, we don't need the strongly generic assumption in some nice cases. And this benefits us a lot.

In the rest of the note, we focus on the uniform subclass $I_{a}\left(f_{1}^{b} \cdots f_{s}^{b}\right)$ under some sufficient generic assumptions. In other words, we don't require it to be $n$-generic, let alone the strong genericness. Notice that in the star configuration case, $b=1$. Therefore, we can also call it the defining ideal of generalized star configuration of hypersurfaces. Whence, the aforementioned information of the projective dimension will play an important role. Recall that we already have a primary-decomposition type formula for the saturation of $I_{a}\left(f_{1}^{b} \cdots f_{s}^{b}\right)$. After sealing these two parts together, we can handle the associated primes of $I_{a}\left(f_{1}^{b} \cdots f_{s}^{b}\right)$ more accurately. In particular, we will feel more at ease when manipulating the symbolic powers of $I_{a}\left(f_{1}^{b} \cdots f_{s}^{b}\right)$.

Recall that if $I$ is a homogeneous ideal of a Noetherian domain $R$, for every positive integer $m$, the $m$-th symbolic power of $I$ is the homogeneous ideal

$$
I^{(m)}:=\bigcap_{\mathfrak{p} \in \operatorname{Ass}(R / I)} I^{m} R_{\mathfrak{p}} \cap R .
$$

For instance, when $I$ is the defining ideal of a reduced affine scheme over an algebraically closed field of characteristic zero, Zariski and Nagata showed that $I^{(m)}$ is generated by the polynomials whose partial derivatives of orders up to $m-1$ vanish on this scheme; c.f. [9]. The research of related topics has continuously attracted the eyes of many researchers; see for instance the recent survey [8] and the references therein.

Regarding symbolic powers, the two constants that we are mostly interested in here are the resurgence and the symbolic defect. Recall that the resurgence of $I$ is defined to be

$$
\rho(I):=\sup \left\{\frac{m}{r}: I^{(m)} \nsubseteq I^{r}\right\} .
$$

It was pointed out in [13] that there are very few results determining the resurgence of the ideal of a subscheme whose dimension is at least one and whose codimension is at least two, apart from ideal of cones and certain monomial ideals.

Meanwhile, the symbolic defect of $I$, as the first estimate of the size of the symbolic power, is defined to be

$$
\operatorname{sdefect}(I, m):=\mu\left(I^{(m)} / I^{m}\right)
$$

for each positive integer $m$. Here, $\mu$ gives the minimal number of generators of the corresponding graded module. Symbolic defect of star configurations has recently been studied by [3], [10] and [18], to name a few. 
Based on the foundation laid above, we are able to scrutinize the symbolic powers of $I_{a}\left(f_{1}^{b} \cdots f_{s}^{b}\right)$ when $a$ is large. As an application, we give closed formulas for the resurgence and symbolic defects in Theorem 4.12. If paying more attention to these two quantities, then one realizes that a delightful description of a minimal generating set of the symbolic powers of $I_{a}\left(f_{1}^{b} \cdots f_{s}^{b}\right)$ is inevitable. This is accomplished in Proposition 4.11, It is worth pointing out here that the corresponding part in [13] depends heavily on the application of the basic double $G$-linkage technique, which requires the Cohen-Macaulay condition. But the latter is not desirable here in general. And this embodies the second main contribution of this note.

For more general $a$, the uniform $a$-product ideal $I_{a}\left(f_{1}^{b} \cdots f_{s}^{b}\right)$ will not be saturated. Whence, the symbolic powers coincide with corresponding standard powers, making the direct probe of this topic dull. Therefore, we take a different path. Notice that there is another algebraic approach in the literature for treating symbolic objects, namely, one only takes intersections over minimal primes of $I$ on the right side of (44); see for instance [25. Definition 4.3.22]. To avoid confusion in notation, we will call it the $m$-th $*$-symbolic power of $I$, namely, we will define

$$
I^{(m)_{*}}:=\bigcap_{\mathfrak{p} \in \operatorname{Min}(I)}\left(I^{m} R_{\mathfrak{p}} \cap R\right) .
$$

Numous studies were also devoted to the symbolic powers along this line; see, for instance, the recent papers [15] and [20].

Regarding symbolic powers of this flavor, the terminologies of *-resurgence and *symbolic defect emerge naturally as well. In contrast, the argument involved for determining these two constants is similar, but more direct. And the outcome is included in the last section of this paper. As an unexpected harvest, the $*$-symbolic defect encodes information regarding whether $I_{a}\left(f_{1}^{b} \cdots f_{s}^{b}\right)$ is a power of the star configuration ideal $I_{c, \mathcal{F}}$ for some $c$; see Proposition 5.6 .

We also want to point out in the end of this section that Conca and Tsakiris [7] recently considered the ideal of subspace arrangements, which is an ideal of fold product of different flavor. Under some generic condition, the ideal they considered can also be linked to discrete polymatroids. In particular, the ideal will have linear quotients.

\section{GENERIC CASE}

The main result of this section is the primary-decomposition type formula of the $a$-fold product ideal $I_{a}\left(f_{1}^{m_{1}} \cdots f_{s}^{m_{s}}\right)$ up to saturation in Theorem 2.4. This result has two happy consequences. Firstly, if the $a$-fold product ideal is a priori saturated, then it leads to the expected formula that we are seeking. Secondly, if the configuration is uniform in the sense that $m_{1}=\cdots=m_{s}$, then it paves the path for our exploration of the symbolic powers of this $a$-fold product ideal. Both are needed in later sections.

Remark 2.1. Throughout this note, we will make substantial use of the following facts.

(a) Let $T=\mathbb{K}\left[z_{1}, \ldots, z_{s}\right]$ and $R=\mathbb{K}\left[x_{0}, \ldots, x_{n}\right]$ be two polynomial rings over a field $\mathbb{K}$. Let $f_{1}, \ldots, f_{s}$ be an $R$-regular sequence of homogeneous elements. Define a homomorphism $\varphi: T \rightarrow R$ induced by $\varphi\left(z_{i}\right)=f_{i}$ for $1 \leq i \leq s$. Then $\varphi$ is flat. Furthermore, if $I$ is a monomial ideal in $T$ and $\boldsymbol{F}$. is a graded minimal free resolution of $T / I$ over $T$. Then $\boldsymbol{F}_{\bullet} \otimes R$ is a graded minimal free resolution of $R / \varphi(I)$ over $R$. In particular, $I$ and the ideal $\varphi(I)$ have the same graded Betti numbers over $T$ and $R$ respectively, 
except possibly with shifts which depend on the degrees of the $f_{i}$. This fact was stated in [13, Lemma 3.1] and its proof.

(b) If a homomorphism $\varphi: T \rightarrow R$ between Noetherian rings is flat, then for arbitrary ideals $I_{1}, I_{2}$ in $T$, we will have $\varphi\left(I_{1} \cap I_{2}\right)=\varphi\left(I_{1}\right) \cap \varphi\left(I_{2}\right)$ as well as $\varphi\left(I_{1}: I_{2}\right)=\varphi\left(I_{1}\right)$ : $\varphi\left(I_{2}\right)$, by [19, Theorem 7.4].

To begin our voyage of generalized star configuration of hypersurfaces, we first notice that both Question 1.4 and Question 1.5 have positive answers for monomial configurations, i.e., when $\mathcal{F}$ is a set of ring variables. Indeed, we have

Lemma 2.2. If $f_{1}, \ldots, f_{s}$ is an $R$-regular sequence of homogeneous elements in $R=$ $\mathbb{K}\left[x_{0}, \ldots, x_{n}\right]$, then

$$
I_{a}\left(f_{1}^{m_{1}} \cdots f_{s}^{m_{s}}\right)=\bigcap_{c=1}^{s}\left(\bigcap_{1 \leq i_{1}<\cdots<i_{c} \leq s}\left\langle f_{i_{1}}, \ldots, f_{i_{c}}\right\rangle^{\mu_{a}\left(i_{1}, \ldots, i_{c}\right)}\right)
$$

and

$$
I_{a+1}\left(f_{1}^{m_{1}} \cdots f_{s-1}^{m_{s-1}} f_{s}^{m_{s}+1}\right): f_{s}=I_{a}\left(f_{1}^{m_{1}} \cdots f_{s-1}^{m_{s-1}} f_{s}^{m_{s}}\right) .
$$

Furthermore, the ideal $I_{a}\left(f_{1}^{m_{1}} \cdots f_{s}^{m_{s}}\right)$ has a Koszul stranded Betti table.

Recall that the notation $\mu_{a}\left(i_{1}, \ldots, i_{c}\right)$ was explained previously in (3). And the assumption of $s \geq n+1$ is not required here.

Proof. The statements hold when $f_{1}, \ldots, f_{s}$ is the sequence of variables $x_{0}, \ldots, x_{s-1}$; see for instance [24, Theorem 2.3 and Corollary 2.4] and their proofs.

For the general case, consider the following treatment as in [13]. Let $T=\mathbb{K}\left[z_{1}, \ldots, z_{s}\right]$ and consider the ring homomorphism

$$
\varphi: T \rightarrow R, \quad z_{i} \mapsto f_{i} .
$$

As $\varphi$ is flat, it remains to apply the facts mentioned in Remark 2.1,

Lemma 2.3. Under the Setting 1.1, we have

$$
I_{a}\left(f_{1}^{m_{1}} \cdots f_{s}^{m_{s}}\right) \subseteq \bigcap_{c=1}^{n}\left(\bigcap_{1 \leq i_{1}<\cdots<i_{c} \leq s}\left\langle f_{i_{1}}, \ldots, f_{i_{c}}\right\rangle^{\mu_{a}\left(i_{1}, \ldots, i_{c}\right)}\right) .
$$

Proof. It is not difficult to argue as in the proof of [24, Lemma 2.1].

Theorem 2.4. Under the Setting 1.1, the equality in Question 1.5 holds up to saturation, i.e.,

$$
I_{a}\left(f_{1}^{m_{1}} \cdots f_{s}^{m_{s}}\right)^{\mathrm{sat}}=\bigcap_{c=1}^{n}\left(\bigcap_{1 \leq i_{1}<\cdots<i_{c} \leq s}\left\langle f_{i_{1}}, \ldots, f_{i_{c}}\right\rangle^{\mu_{a}\left(i_{1}, \ldots, i_{c}\right)}\right) .
$$

Proof. We will follow the technique in the proofs of [12, Corollary 4.9] and 24, Proposition 2.2]. Regarding the equality in (5), we have at least the containment LHS $\subseteq$ RHS, by Lemma 2.3. Thus, it suffices to take any graded non-maximal ideal $\mathfrak{p}$ containing $I_{a}\left(f_{1}^{m_{1}} \cdots f_{s}^{m_{s}}\right)$ and show that the equality in (5) holds locally with respect to $\mathfrak{p}$.

First of all, one can verify with ease that

$$
I_{a}\left(f_{1}^{m_{1}} \cdots f_{s}^{m_{s}}\right)=\sum_{k=0}^{m_{s}} f_{s}^{k} I_{a-k}\left(f_{1}^{m_{1}} \cdots f_{s-1}^{m_{s-1}}\right) .
$$


Since

$$
I_{a-m_{s}}\left(f_{1}^{m_{1}} \cdots f_{s-1}^{m_{s-1}}\right) \supseteq \cdots \supseteq I_{a-k}\left(f_{1}^{m_{1}} \cdots f_{s-1}^{m_{s-1}}\right) \supseteq \cdots \supseteq I_{a}\left(f_{1}^{m_{1}} \cdots f_{s-1}^{m_{s-1}}\right)
$$

for $0 \leq k \leq m_{s}$, we will have

$$
I_{a}\left(f_{1}^{m_{1}} \cdots f_{s}^{m_{s}}\right) R_{\mathfrak{p}}=I_{a-m_{s}}\left(f_{1}^{m_{1}} \cdots f_{s-1}^{m_{s-1}}\right) R_{\mathfrak{p}},
$$

once $f_{s} \notin \mathfrak{p}$.

Now, without loss of generality, we may assume that $f_{1}, \ldots, f_{r} \in \mathfrak{p}$ while $f_{r+1}, \ldots, f_{s} \notin \mathfrak{p}$. Because of the generic assumption on $\mathcal{F}$, we have that $r \leq h t(\mathfrak{p})<\operatorname{ht}(\mathfrak{m})=n+1$. It is clear that

$$
\bigcap_{c=1}^{\min (s, n)}\left(\bigcap_{1 \leq i_{1}<\cdots<i_{c} \leq s}\left\langle f_{i_{1}}, \ldots, f_{i_{c}}\right\rangle^{\mu_{a}\left(i_{1}, \ldots, i_{c}\right)}\right) R_{\mathfrak{p}}=\bigcap_{c=1}^{r}\left(\bigcap_{1 \leq i_{1}<\cdots<i_{c} \leq r}\left\langle f_{i_{1}}, \ldots, f_{i_{c}}\right\rangle^{\mu_{a}\left(i_{1}, \ldots, i_{c}\right)}\right) R_{\mathfrak{p}},
$$

while

$$
I_{a}\left(f_{1}^{m_{1}} \cdots f_{s}^{m_{s}}\right)^{\mathrm{sat}} R_{\mathfrak{p}}=I_{a}\left(f_{1}^{m_{1}} \cdots f_{s}^{m_{s}}\right) R_{\mathfrak{p}}=I_{a-\sum_{c=r+1}^{s} m_{c}}\left(f_{1}^{m_{1}} \cdots f_{r}^{m_{r}}\right) R_{\mathfrak{p}}
$$

by applying the equality in (6) repeatedly.

As the last step, it remains to verify that

$$
I_{a-\sum_{c=r+1}^{s} m_{c}}\left(f_{1}^{m_{1}} \cdots f_{r}^{m_{r}}\right)=\bigcap_{c=1}^{r}\left(\bigcap_{1 \leq i_{1}<\cdots<i_{c} \leq r}\left\langle f_{i_{1}}, \ldots, f_{i_{c}}\right\rangle^{\mu_{a}\left(i_{1}, \ldots, i_{c}\right)}\right) .
$$

But this is exactly what we have shown in Lemma 2.2 .

The decomposition in (5) can be refined once we know more about the associated primes of $I_{a}\left(f_{1}^{m_{1}} \cdots f_{s}^{m_{s}}\right)$.

Theorem 2.5. Let $\mathcal{F}=\left\{f_{1}, \ldots, f_{s}\right\}$ be a set of $\hat{c}$-generic forms in $R=\mathbb{K}\left[x_{0}, \ldots, x_{n}\right]$. Let $a, m_{1}, \ldots, m_{s}$ be positive integers with $a \leq m_{1}+\cdots+m_{s}$. Suppose that $\mathrm{ht}(\mathfrak{q}) \leq \hat{c} \leq n$ for each $\mathfrak{q} \in \operatorname{Ass}\left(R / I_{a}\left(f_{1}^{m_{1}} \cdots f_{s}^{m_{s}}\right)\right)$. Then we have

$$
I_{a}\left(f_{1}^{m_{1}} \cdots f_{s}^{m_{s}}\right)=\bigcap_{c=1}^{\hat{c}}\left(\bigcap_{1 \leq i_{1}<\cdots<i_{c} \leq s}\left\langle f_{i_{1}}, \ldots, f_{i_{c}}\right\rangle^{\mu_{a}\left(i_{1}, \ldots, i_{c}\right)}\right) .
$$

Proof. The proof is similar to that of Theorem 2.4. And one only needs to take prime ideals $\mathfrak{p}$ of $R$ such that $h t(\mathfrak{p}) \leq \hat{c}$, by our assumption.

Remark 2.6. It follows from [6, Proposition 1.2.13 and Theorem 1.3.3] that ht $(\mathfrak{q}) \leq$ $\operatorname{proj} \operatorname{dim}\left(R / I_{a}\left(f_{1}^{m_{1}} \cdots f_{s}^{m_{s}}\right)\right)$ for each $\mathfrak{q} \in \operatorname{Ass}\left(R / I_{a}\left(f_{1}^{m_{1}} \cdots f_{s}^{m_{s}}\right)\right)$. Therefore, to apply Theorem 2.5 efficiently, one needs to bound the projective dimension of $I_{a}\left(f_{1}^{m_{1}} \cdots f_{s}^{m_{s}}\right)$.

Now, consider the defining ideal of the star configuration of hypersurfaces in $\mathbb{P}^{n}$

$$
I_{c, \mathcal{F}}:=\bigcap_{1 \leq i_{1}<\cdots<i_{c} \leq s}\left\langle f_{i_{1}}, \ldots, f_{i_{c}}\right\rangle .
$$

It was shown in [13, Theorem 3.3] that

$$
I_{c, \mathcal{F}}=I_{s-c+1}\left(f_{1} \cdots f_{s}\right) \text {. }
$$

Indeed, it was shown in [13, Theorem 3.6] that for each positive integer $m$, the symbolic power

$$
I_{c, \mathcal{F}}^{(m)}=\bigcap_{1 \leq i_{1}<\cdots<i_{c} \leq s}\left\langle f_{i_{1}}, \ldots, f_{i_{c}}\right\rangle^{m}
$$


Meanwhile, it is clear that

$$
I_{a}\left(f_{1}^{m_{1}} \cdots f_{s}^{m_{s}}\right)^{m}=I_{m a}\left(f_{1}^{m m_{1}} \cdots f_{s}^{m m_{s}}\right)
$$

for each positive integer $m$. Consequently,

$$
I_{c, \mathcal{F}}^{m}=\left(I_{s-c+1}\left(f_{1} \cdots f_{s}\right)\right)^{m}=I_{m(s-c+1)}\left(f_{1}^{m} \cdots f_{s}^{m}\right) .
$$

Therefore, we obtain the following corollary from Theorem 2.4 .

Corollary 2.7. Under the Setting 1.1, the saturation of the power of the star configuration ideal can be expressed as

$$
\left(I_{c, \mathcal{F}}^{m}\right)^{\mathrm{sat}}=I_{c, \mathcal{F}}^{(m)} \cap I_{c+1, \mathcal{F}}^{(2 m)} \cap \cdots \cap I_{n, \mathcal{F}}^{((n-c+1) m)},
$$

or, equivalently,

$$
\left(I_{s-c+1}\left(f_{1} \cdots f_{s}\right)^{m}\right)^{\mathrm{sat}}=\bigcap_{c \leq k \leq n} I_{s-k+1}\left(f_{1} \cdots f_{s}\right)^{((k-c+1) m)} .
$$

Indeed, the latest equality can be easily generalized by considering a similar decomposition of $I_{a}\left(f_{1}^{b} \cdots f_{s}^{b}\right)^{m}=I_{m a}\left(f_{1}^{m b} f_{2}^{m b} \cdots f_{s}^{m b}\right)$ of positive dimension, for positive integers $a, b$ and $m$. To facilitate discussions in later sections, we will fix the following notations.

Setting 2.8. Let $a, b, c_{0}, n, s, \mu_{a}^{0}$ be positive integers such that

$$
c_{0}:=s-\left\lfloor\frac{a-1}{b}\right\rfloor, \quad \mu_{a}^{0}:=a-b\left(s-c_{0}\right), \quad s \geq n+1 \quad \text { and } \quad b(s-n)+1 \leq a \leq s b .
$$

Here, we explain a little bit the origin of the notations chosen above. Under such setting, for each component $\left\langle f_{i_{1}}, \ldots, f_{i_{c}}\right\rangle$ in (5) with $m_{1}=\cdots=m_{s}=b$, the exponent $\mu_{a}\left(i_{1}, \ldots, i_{c}\right)=a-b(s-c) \geq 1$ precisely when $c \geq s-\left\lfloor\frac{a-1}{b}\right\rfloor=c_{0}$. Meanwhile,

$$
\mu_{a}^{0}=a-b\left(s-c_{0}\right)=a-b\left\lfloor\frac{a-1}{b}\right\rfloor \in\{1,2, \ldots, b\} .
$$

Whence, the exponent in (5) satisfies

$$
\mu_{a}\left(i_{1}, \ldots, i_{c}\right)=a-b(s-c)=a-b\left(s-c_{0}\right)+b\left(c-c_{0}\right)=\mu_{a}^{0}+b\left(c-c_{0}\right)
$$

for $c_{0} \leq c \leq s$. In order that $I_{a}\left(f_{1}^{b} \cdots f_{s}^{b}\right)$ is not zero dimensional, we have to restrict ourselves to those $a$ 's such that $c_{0} \leq n$, i.e., $a \geq b(s-n)+1$. To wrap up this short discussion, we remark that

$$
a=b\left(s-c_{0}\right)+\mu_{a}^{0} \quad \text { with } \quad 1 \leq \mu_{a}^{0} \leq b .
$$

This non-standard long division might be more intuitive for some readers when verifying various estimates and equalities in later sections.

Now, it follows directly from Theorem 2.4 and the equality (8) that we have the following formula for the uniform a-fold product ideal $I_{a}\left(\mathcal{F}^{b}\right):=I_{a}\left(f_{1}^{b} \cdots f_{s}^{b}\right)$ in $R=\mathbb{K}\left[x_{0}, \ldots, x_{n}\right]$. We also call it the defining ideal of generalized star configuration of hypersurfaces.

Corollary 2.9. With the assumptions in Settings 1.1 and 2.8, we have

$$
\left(I_{a}\left(\mathcal{F}^{b}\right)^{m}\right)^{\mathrm{sat}}=\bigcap_{c_{0} \leq c \leq n} I_{c, \mathcal{F}}^{\left(m\left(\mu_{a}^{0}+b\left(c-c_{0}\right)\right)\right)}
$$

for each positive integer $m$. 


\section{Strongly Generic CASE}

Unfortunately, but not surprisingly, a computation by the software Macaulay2 [14] shows that all of Question 1.3. Question 1.4 and Question 1.5 have negative answers in general for quadratic generic forms in $\mathbb{K}\left[x_{0}, x_{1}, x_{2}\right]$.

Thus, in the following, due to the above technical obstruction, we have to inflict a stricter condition so that the forms we treat are essentially linear. Suppose first that the forms in $\mathcal{F}=\left\{f_{1}, \ldots, f_{s}\right\} \subset R=\mathbb{K}\left[x_{0}, \ldots, x_{n}\right]$ have a common degree $d$. Then, we say that $\mathcal{F}$ is strongly generic if it is generic and

$$
\operatorname{dim}_{\mathbb{K}}\left(\mathbb{K} f_{1}+\cdots+\mathbb{K} f_{s}\right)=n+1=\operatorname{dim}(R) .
$$

Notice that for every $k \geq n+1$, we will have the ideals

$$
\left\langle f_{1}, \ldots, f_{n+1}\right\rangle=\left\langle f_{i_{1}}, \ldots, f_{i_{k}}\right\rangle \quad \text { for any } 1 \leq i_{1}<\cdots<i_{k} \leq s .
$$

This common $\mathfrak{m}$-primary complete intersection ideal will be denoted by $\mathfrak{m}_{\mathcal{F}}$.

Remark 3.1. When $d=1$, then the set $\mathcal{F}$ being strongly generic is equivalent to it being generic.

In this section, we will shift our focus to $a$-fold product ideals of strongly generic sets of forms. As a warm-up, we first notice that Lemma 2.2 can be strengthened and all of Question 1.3, Question 1.4 and Question 1.5 have positive answers in this situation.

Proposition 3.2. Under the Setting 1.1, suppose in addition that $\mathcal{F}=\left\{f_{1}, \ldots, f_{s}\right\}$ is strongly generic. Then,

$$
I_{a}\left(f_{1}^{m_{1}} \cdots f_{s}^{m_{s}}\right)=\bigcap_{c=1}^{s}\left(\bigcap_{1 \leq i_{1}<\cdots<i_{c} \leq s}\left\langle f_{i_{1}}, \ldots, f_{i_{c}}\right\rangle^{\mu_{a}\left(i_{1}, \ldots, i_{c}\right)}\right)
$$

and

$$
I_{a+1}\left(f_{1}^{m_{1}} \cdots f_{s-1}^{m_{s-1}} f_{s}^{m_{s}+1}\right): f_{s}=I_{a}\left(f_{1}^{m_{1}} \cdots f_{s-1}^{m_{s-1}} f_{s}^{m_{s}}\right) .
$$

Furthermore, the ideal $I_{a}\left(f_{1}^{m_{1}} \cdots f_{s}^{m_{s}}\right)$ has a Koszul stranded Betti table.

Proof. By virtue of Lemma 2.2, it suffices to consider the case when $s \geq n+2$. Whence, under the assumptions on $\mathcal{F}$, for each $j=n+2, \ldots, s$, we will have $f_{j}=\sum_{i=1}^{n+1} \mu_{j i} f_{i}$ for some $\mu_{j i} \in \mathbb{K}$. Now consider a new configuration $\mathcal{L}=\left\{\ell_{1}, \ldots, \ell_{s}\right\}$ in $S=\mathbb{K}\left[y_{1}, \ldots, y_{n+1}\right]$, where $\ell_{1}=y_{1}, \ldots, \ell_{n+1}=y_{n+1}$, and $\ell_{j}=\sum_{i=1}^{n+1} \mu_{j i} y_{i}$ for $j=n+2, \ldots, s$. Since $\mathcal{F}$ is strongly generic, so is $\mathcal{L}$. Meanwhile, the desired properties hold if we replace $\mathcal{F}$ by $\mathcal{L}$, because of [24, Theorem 2.3 and Corollary 2.4] and their proofs.

To finish our proof, consider the homomorphism $\psi: S \rightarrow R$ induced by $\psi\left(y_{i}\right)=f_{i}$ for $1 \leq i \leq n+1$. As $f_{1}, \ldots, f_{n+1} \in R$ form a regular sequence, the map $\psi$ is flat. Notice that $I_{a}\left(f_{1}^{m_{1}} \cdots f_{s}^{m_{s}}\right)=\psi\left(I_{a}\left(\ell_{1}^{m_{1}} \cdots \ell_{s}^{m_{s}}\right)\right)$. Now, it remains to apply the facts stated in Remark 2.1.

Recall that the following formula was first conjectured by Geramita, Harbourne and Migliore in [12, Conjecture 4.1] for any generic set $\mathcal{F}$ of linear forms. It was later established by Tohăneanu and Xie in [24, Theorem 3.2].

$$
I_{c, \mathcal{F}}^{m}=I_{c, \mathcal{F}}^{(m)} \cap I_{c+1, \mathcal{F}}^{(2 m)} \cap \cdots \cap I_{n, \mathcal{F}}^{((n-c+1) m)} \cap \mathfrak{m}^{(s-c+1) m} .
$$

Once we apply the flat argument in the proof of Proposition 3.2 to the above formula, we immediately obtain the following result. 
Proposition 3.3. Under the Setting 1.1, suppose in addition that $\mathcal{F}=\left\{f_{1}, \ldots, f_{s}\right\}$ is strongly generic. Then the power of the star configuration ideal can be expressed as

$$
I_{c, \mathcal{F}}^{m}=I_{c, \mathcal{F}}^{(m)} \cap I_{c+1, \mathcal{F}}^{(2 m)} \cap \cdots \cap I_{n, \mathcal{F}}^{((n-c+1) m)} \cap \mathfrak{m}_{\mathcal{F}}^{(s-c+1) m} .
$$

Proof. It remains to point out the formula in (8) regarding the symbolic powers.

The Koszul stranded Betti table part in Proposition 3.2 can be strengthened. Notice that as a generalization of ideals with linear quotients, Mantero introduced the notion of complete intersection quotients in [18, Definition 6.1].

Definition 3.4. Let $I$ be a homogeneous ideal in the polynomial ring $R=\mathbb{K}\left[x_{0}, \ldots, x_{n}\right]$. We say that $I$ has c.i. quotients if there exists a total order $h_{1}>\cdots>h_{r}$ on a generating set $\left\{h_{1}, \ldots, h_{r}\right\}$ of $I$, such that for any $1 \leq i \leq r-1$, the colon ideal $\left\langle h_{1}, \ldots, h_{i}\right\rangle: h_{i+1}$ is a complete intersection ideal. Furthermore, if each such colon ideal has the same degree $d$, then we say that $I$ has $d$-c.i. quotients.

It is clear that $I$ has linear quotients precisely when $I$ has 1-c.i. quotients. Furthermore, if $I$ has $d$-c.i. quotients, then it has a Koszul stranded Betti table by [18, Corollary 6.6].

The following result is crucial for our voyage of generalized star configurations.

Lemma 3.5. Let $I$ be a monomial ideal having linear quotients in $T=\mathbb{K}\left[z_{1}, \ldots, z_{s}\right]$ and $\mathcal{F}=\left\{f_{1}, \ldots, f_{s}\right\}$ a strongly generic set of $d$-forms in $R=\mathbb{K}\left[x_{0}, \ldots, x_{n}\right]$. Regarding the homomorphism $\varphi: T \rightarrow R$ induced by $z_{i} \mapsto f_{i}$ for each $i$, the specialization $\varphi(I)$ has d-c.i. quotients.

Proof. Since $I$ is monomial and has linear quotients, there is a total order $\boldsymbol{z}^{\boldsymbol{\beta}_{1}}>\cdots>\boldsymbol{z}^{\boldsymbol{\beta}_{r}}$ on the minimal monomial generating set $\left\{\boldsymbol{z}^{\boldsymbol{\beta}_{1}}, \ldots, \boldsymbol{z}^{\boldsymbol{\beta}_{r}}\right\}$ of $I$ so that each successive colon ideal $\left\langle\boldsymbol{z}^{\boldsymbol{\beta}_{1}}, \ldots, \boldsymbol{z}^{\boldsymbol{\beta}_{i}}\right\rangle: \boldsymbol{z}^{\boldsymbol{\beta}_{i+1}}$ is generated by a subset of the variables $\left\{z_{1}, \ldots, z_{s}\right\}$.

Now, $\varphi(I)=\left\langle\varphi\left(\boldsymbol{z}^{\beta_{1}}\right), \ldots, \varphi\left(\boldsymbol{z}^{\boldsymbol{\beta}_{r}}\right)\right\rangle$. For each $i \leq r-1$, if $\varphi\left(\boldsymbol{z}^{\boldsymbol{\beta}_{i+1}}\right) \notin \varphi\left(\left\langle\boldsymbol{z}^{\boldsymbol{\beta}_{1}}, \ldots, \boldsymbol{z}^{\boldsymbol{\beta}_{i}}\right\rangle\right)$, we claim that

$$
\varphi\left(\left\langle\boldsymbol{z}^{\boldsymbol{\beta}_{1}}, \ldots, \boldsymbol{z}^{\boldsymbol{\beta}_{i}}\right\rangle\right): \varphi\left(\boldsymbol{z}^{\boldsymbol{\beta}_{i+1}}\right)=\varphi\left(\left\langle\boldsymbol{z}^{\boldsymbol{\beta}_{1}}, \ldots, \boldsymbol{z}^{\boldsymbol{\beta}_{i}}\right\rangle: \boldsymbol{z}^{\boldsymbol{\beta}_{i+1}}\right) .
$$

Of course, if $\varphi\left(\boldsymbol{z}^{\boldsymbol{\beta}_{i+1}}\right) \in \varphi\left(\left\langle\boldsymbol{z}^{\boldsymbol{\beta}_{1}}, \ldots, \boldsymbol{z}^{\boldsymbol{\beta}_{i}}\right\rangle\right)$, then we can simply remove $\varphi\left(\boldsymbol{z}^{\boldsymbol{\beta}_{i+1}}\right)$ from the generating set of $\varphi(I)$.

For simplicity, for this fixed $i$, suppose that the quotient ideal $\left\langle\boldsymbol{z}^{\boldsymbol{\beta}_{1}}, \ldots, \boldsymbol{z}^{\boldsymbol{\beta}_{i}}\right\rangle: \boldsymbol{z}^{\boldsymbol{\beta}_{i+1}}$ is generated by $z_{j_{1}}, z_{j_{2}}, \ldots, z_{j_{t}}$ with $1 \leq j_{1}<j_{2}<\cdots<j_{t} \leq s$. Hence, the aforementioned claim asks for

$$
\left\langle\varphi\left(\boldsymbol{z}^{\boldsymbol{\beta}_{1}}\right), \ldots, \varphi\left(\boldsymbol{z}^{\boldsymbol{\beta}_{i}}\right)\right\rangle: \varphi\left(\boldsymbol{z}^{\boldsymbol{\beta}_{i+1}}\right)=\varphi\left(\left\langle z_{j_{1}}, \ldots, z_{j_{t}}\right\rangle\right) .
$$

Regarding the latest equality, as the containment LHS $\supseteq$ RHS is clear, it remains to prove the reverse containment

$$
\left\langle\varphi\left(\boldsymbol{z}^{\boldsymbol{\beta}_{1}}\right), \ldots, \varphi\left(\boldsymbol{z}^{\boldsymbol{\beta}_{i}}\right)\right\rangle: \varphi\left(\boldsymbol{z}^{\boldsymbol{\beta}_{i+1}}\right) \subseteq \varphi\left(\left\langle z_{j_{1}}, \ldots, z_{j_{t}}\right\rangle\right) .
$$

(a) When $t \geq n+1$, then the RHS of (12) is simply $\mathfrak{m}_{\mathcal{F}}$. We will use the notations in the proof of Proposition [3.2. Whence, there exists forms $g_{1}, \ldots, g_{r} \in S=$ $\mathbb{K}\left[y_{1}, \ldots, y_{n+1}\right]$ such that $\varphi\left(\boldsymbol{z}^{\boldsymbol{\beta}_{j}}\right)=\psi\left(g_{j}\right)$ for each $j$. As $\varphi\left(\boldsymbol{z}^{\boldsymbol{\beta}_{i+1}}\right) \notin \varphi\left(\left\langle\boldsymbol{z}^{\boldsymbol{\beta}_{1}}, \ldots, \boldsymbol{z}^{\boldsymbol{\beta}_{i}}\right\rangle\right)$, $g_{i+1} \notin\left\langle g_{1}, \ldots, g_{i}\right\rangle$. Therefore, $\left\langle g_{1}, \ldots, g_{i}\right\rangle: g_{i+1} \subseteq\left\langle y_{1}, \ldots, y_{n+1}\right\rangle$, the graded maximal ideal of $S$. Since $\psi$ is flat, this implies that

$$
\psi\left(\left\langle g_{1}, \ldots, g_{i}\right\rangle\right): \psi\left(g_{i+1}\right)=\psi\left(\left\langle g_{1}, \ldots, g_{i}\right\rangle: g_{i+1}\right) \subseteq \psi\left(\left\langle y_{1}, \ldots, y_{n+1}\right\rangle\right)=\mathfrak{m}_{\mathcal{F}} .
$$

Hence the containment (12) holds in this case. 
(b) When $t \leq n$, to prove (12), it suffices to prove

$$
\varphi\left(\boldsymbol{z}^{\boldsymbol{\beta}_{k}}\right): \varphi\left(\boldsymbol{z}^{\boldsymbol{\beta}_{i+1}}\right) \subseteq\left\langle f_{j_{1}}, \ldots, f_{j_{t}}\right\rangle
$$

for $k=1, \ldots, i$, by applying [18, Proposition 6.11] with $c=n$. Notice that as $\boldsymbol{z}^{\boldsymbol{\beta}_{k}}: \boldsymbol{z}^{\boldsymbol{\beta}_{i+1}} \subseteq\left\langle z_{j_{1}}, \ldots, z_{j_{t}}\right\rangle$,

$$
\operatorname{supp}\left(\boldsymbol{z}^{\boldsymbol{\beta}_{k}} / \operatorname{gcd}\left(\boldsymbol{z}^{\boldsymbol{\beta}_{k}}, \boldsymbol{z}^{\boldsymbol{\beta}_{i+1}}\right)\right) \cap\left\{j_{1}, \ldots, j_{t}\right\} \neq \varnothing .
$$

Without loss of generality, say $j_{1}$ belongs to this intersection. Then,

$$
\begin{aligned}
\varphi\left(\boldsymbol{z}^{\boldsymbol{\beta}_{k}}\right): \varphi\left(\boldsymbol{z}^{\boldsymbol{\beta}_{i+1}}\right) & =\varphi\left(\boldsymbol{z}^{\boldsymbol{\beta}_{k}} / \operatorname{gcd}\left(\boldsymbol{z}^{\boldsymbol{\beta}_{k}}, \boldsymbol{z}^{\boldsymbol{\beta}_{i+1}}\right)\right): \varphi\left(\boldsymbol{z}^{\boldsymbol{\beta}_{i+1}} / \operatorname{gcd}\left(\boldsymbol{z}^{\boldsymbol{\beta}_{k}}, \boldsymbol{z}^{\boldsymbol{\beta}_{i+1}}\right)\right) \\
& \subseteq\left\langle f_{j_{1}}\right\rangle \subseteq\left\langle f_{j_{1}}, \ldots, f_{j_{t}}\right\rangle,
\end{aligned}
$$

where the first containment is due to [18, Lemma 6.8]. Since this confirms the containment in (13), our proof is finished.

It is clear from the above proof that the additional strongly generic assumption is only needed when dealing with the maximal part $\mathfrak{m}_{\mathcal{F}}$. Thus, we will have a variant result under the mild generic condition.

Lemma 3.6. Let $I$ be a monomial ideal having linear quotients in $T=\mathbb{K}\left[z_{1}, \ldots, z_{s}\right]$ and $\mathcal{F}=\left\{f_{1}, \ldots, f_{s}\right\}$ a set of c-generic forms in $R=\mathbb{K}\left[x_{0}, \ldots, x_{n}\right]$. Suppose that $\operatorname{proj}^{\operatorname{dim}_{T}}(I) \leq$ $c<\operatorname{dim}(R)=n+1$. Regarding the homomorphism $\varphi: T \rightarrow R$ induced by $z_{i} \mapsto f_{i}$ for each $i$, the specialization $\varphi(I)$ has c.i. quotients. If in addition all forms in $\mathcal{F}$ are of degree $d$, then $\varphi(I)$ has d-c.i. quotients.

Proof. It suffices to point out that

$$
\operatorname{proj} \operatorname{dim}_{T}(I)=\max \left\{\mu\left(\left\langle\boldsymbol{z}^{\boldsymbol{\beta}_{1}}, \ldots, \boldsymbol{z}^{\boldsymbol{\beta}_{i}}\right\rangle: \boldsymbol{z}^{\boldsymbol{\beta}_{i+1}}\right): 1 \leq i \leq r-1\right\}
$$

where $\mu$ denotes the minimal number of generators. Now, the remaining argument will be similar. We will only encounter the $t \leq c \leq n$ case, where the Lemma 6.8 and Proposition 6.11 of [18] still apply.

With the above preparation, we are ready to state the main result of this section.

Theorem 3.7. Let $R=\mathbb{K}\left[x_{0}, \ldots, x_{n}\right]$ and $T=\mathbb{K}\left[z_{1}, \ldots, z_{s}\right]$ be two polynomial rings over the field $\mathbb{K}$. Let $\mathcal{F}=\left\{f_{1}, \ldots, f_{s}\right\}$ be a set of forms in $R$ and $a, m_{1}, \ldots, m_{s}$ be positive integers with $a \leq m_{1}+\cdots+m_{s}$.

(a) If $\mathcal{F}$ is a strongly generic set of d-forms, then the a-fold product ideal $I_{a}\left(f_{1}^{m_{1}} \cdots f_{s}^{m_{s}}\right)$ has d-c.i. quotients.

(b) If $\operatorname{proj}_{\operatorname{dim}_{T}}\left(I_{a}\left(z_{1}^{m_{1}} \cdots z_{s}^{m_{s}}\right)\right) \leq c \leq n$ and $\mathcal{F}$ is c-generic, then the a-fold product ideal $I_{a}\left(f_{1}^{m_{1}} \cdots f_{s}^{m_{s}}\right)$ has c.i. quotients. If in addition all forms in $\mathcal{F}$ are of degree $d$, then $I_{a}\left(f_{1}^{m_{1}} \cdots f_{s}^{m_{s}}\right)$ has d-c.i. quotients.

Proof. The monomial ideal $I_{a}\left(z_{1}^{m_{1}} \cdots z_{s}^{m_{s}}\right)$ in $T$ is known as the ideal of Veronese type. In particular, it is a polymatroidal ideal and has linear quotients; see [16, Example 12.2.8 and Theorem 12.6.2]. Now, we can apply Lemma 3.5 and Lemma 3.6.

Corollary 3.8. Let $\Sigma:=(\underbrace{f_{1}, \ldots, f_{1}}_{m_{1}}, \ldots, \underbrace{f_{s}, \ldots, f_{s}}_{m_{s}})$ be a collection of linear forms in $\mathbb{K}\left[x_{0}, \ldots, x_{n}\right]$, with $s, m_{1}, \ldots, m_{s} \geq 1$, and with $\operatorname{Supp}(\Sigma)=\left\{f_{1}, \ldots, f_{s}\right\}$ being generic. Then for any $1 \leq a \leq m_{1}+\cdots+m_{s}$, the ideal $I_{a}\left(f_{1}^{m_{1}} f_{2}^{m_{2}} \cdots f_{s}^{m_{s}}\right)$ has linear quotients. 
Proof. When we are dealing with linear forms, the set $\operatorname{Supp}(\Sigma)$ being strongly generic is equivalent to being generic. Now, we apply Theorem 3.7](a).

Since equi-generated ideals having linear quotients will have linear resolutions, we recover the linear resolution result in [24, Theorem 2.3] by the above corollary.

Corollary 3.9. Under the assumptions in Theorem 3.7, suppose in addition that all forms in $\mathcal{F}$ are of degree $d$. Then the Castelnuovo-Mumford regularity

$$
\operatorname{reg}_{R}\left(R / I_{a}\left(f_{1}^{m_{1}} \cdots f_{s}^{m_{s}}\right)\right)=d(a+p-1)-p
$$

for $p=\operatorname{proj} \operatorname{dim}_{R}\left(R / I_{a}\left(f_{1}^{m_{1}} \cdots f_{s}^{m_{s}}\right)\right)$.

Proof. Note that the ideal $I_{a}\left(f_{1}^{m_{1}} \cdots f_{s}^{m_{s}}\right)$ is equi-generated in degree ad. Thus, the largest degree shift of the graded minimal free resolution happens at the top homological degree.

It is noted in [18, Remark 6.4 and Corollary 6.6] that any ideal with $d$-c.i. quotients has a Koszul stranded Betti table, which is completely determined by the sizes of minimal generating sets of the successive colon ideals. To be more precise, suppose that an ideal $I \subset T=\mathbb{K}\left[z_{1}, \ldots, z_{s}\right]$ has $d$-c.i. quotients with respect to a total order on a generating set $G(I)=\left\{g_{1}>\cdots>g_{h}\right\}$. For each $1 \leq i \leq h$, let $r_{k}$ be the minimal number of generators of $\left\langle g_{1}, \ldots, g_{i-1}\right\rangle:\left\langle g_{i}\right\rangle$. Now, $T / I$ has a minimal graded $T$-free resolution $\boldsymbol{F}_{\bullet}=\bigoplus_{i} F_{i}$ with

$$
F_{i}=\bigoplus_{k=1}^{h} T\left(-\left(\operatorname{deg}\left(g_{k}\right)+d(i-1)\right)\right)^{\left(\begin{array}{c}
r_{k} \\
i-1
\end{array}\right)}
$$

for $i \geq 1$.

Back to the specialization discussions in Lemma 3.5 and Lemma 3.6. As the sizes of successive colon ideals won't get larger after specialization by the affiliated proofs, we can compare the Betti numbers of the ideals with ease.

Proposition 3.10. Under the assumptions in Lemma 3.5 or Lemma 3.6, we have

$$
\beta_{i, d j}^{R}(R / \varphi(I)) \leq \beta_{i, j}^{T}(T / I)
$$

for any $i \geq 1$. In particular, for the a-fold product ideal $I_{a}\left(f_{1}^{m_{1}} \cdots f_{s}^{m_{s}}\right)=\varphi\left(I_{a}\left(z_{1}^{m_{1}} \cdots z_{s}^{m_{s}}\right)\right)$ in $R$, if all forms in $\mathcal{F}=\left\{f_{1}, \ldots, f_{s}\right\}$ are of degree $d$, then we have

$$
\beta_{i, d(a+i-1)}^{R}\left(R / I_{a}\left(f_{1}^{m_{1}} \cdots f_{s}^{m_{s}}\right)\right) \leq \beta_{i, a+i-1}^{T}\left(T / I_{a}\left(z_{1}^{m_{1}} \cdots z_{s}^{m_{s}}\right)\right)
$$

and

for each $i \geq 1$.

$$
\beta_{i, j}^{R}\left(R / I_{a}\left(f_{1}^{m_{1}} \cdots f_{s}^{m_{s}}\right)\right)=0 \quad \text { for } j \neq d(a+i-1)
$$

Proof. The two quotient modules $R / \varphi(I)$ and $T / I$ have graded minimal free resolutions $\mathcal{F}_{\bullet}=\bigoplus_{i} F_{i}$ and $\mathcal{F}_{\bullet}^{\prime}=\bigoplus_{i} F_{i}^{\prime}$ respectively such that for each direct summand

$$
R\left(-d\left(\operatorname{deg}\left(\boldsymbol{z}^{\boldsymbol{\beta}_{k}}\right)+(i-1)\right)\right)^{\left(\begin{array}{c}
r_{k} \\
i-1
\end{array}\right)}
$$

of $F_{i}$, there is a corresponding direct summand

$$
T\left(-\left(\operatorname{deg}\left(\boldsymbol{z}^{\boldsymbol{\beta}_{k}}\right)+(i-1)\right)\right)^{\left(\begin{array}{c}
r_{k}^{\prime} \\
i-1
\end{array}\right)}
$$

of $F_{i}^{\prime}$. Furthermore, $r_{k} \leq r_{k}^{\prime}$ for each $k$. The rest of the argument is clear. 
Corollary 3.11. Under the assumptions in Lemma 3.5 or Lemma 3.6, we have

$$
\text { proj } \operatorname{dim}_{R}(R / \varphi(I)) \leq \operatorname{proj} \operatorname{dim}_{T}(T / I) .
$$

Conversely, equality in (15) for $i=2$ will imply that $\mathcal{F}$ actually forms a regular sequence in the zero-dimensional case.

Corollary 3.12. Under the assumptions in Lemma 3.5, suppose in addition that $I \subset T$ is zero-dimensional and equi-generated with $\beta_{2, d(a+1)}^{R}(R / \varphi(I))=\beta_{2, a+1}^{T}(T / I)$, then $f_{1}, \ldots, f_{s}$ is indeed a regular sequence.

Proof. Suppose that in the proof of Lemma 3.5, $\left\langle\boldsymbol{z}^{\boldsymbol{\beta}_{1}}, \ldots, \boldsymbol{z}^{\boldsymbol{\beta}_{i}}\right\rangle: \boldsymbol{z}^{\boldsymbol{\beta}_{i+1}}$ is minimally generated by $r_{i}$ variables for $i=0,1, \ldots, r-1$. Meanwhile, suppose that $\left\langle\varphi\left(\boldsymbol{z}^{\boldsymbol{\beta}_{1}}\right), \ldots, \varphi\left(\boldsymbol{z}^{\boldsymbol{\beta}_{i}}\right)\right\rangle$ : $\varphi\left(\boldsymbol{z}^{\boldsymbol{\beta}_{i+1}}\right)$ is minimally generated by $r_{i}^{\prime}$ forms in $\mathcal{F}$; when this colon ideal is $R$, we will choose $r_{i}^{\prime}=0$. We have mentioned earlier that $r_{i} \geq r_{i}^{\prime}$ for all $i$. It is clear that the two total Betti numbers in the condition are $\sum_{i} r_{i}$ and $\sum_{i} r_{i}^{\prime}$ respectively. Thus, the equality of these two Betti numbers implies that $r_{i}=r_{i}^{\prime}$ for all $i$. But as $T / I$ is zero-dimensional, proj $\operatorname{dim}_{T}(T / I)=\operatorname{dim}(T)$. Thus, by applying (14), one has

$$
r_{i_{0}}=\operatorname{dim}(T) \geq \operatorname{dim}(R) \geq r_{i_{0}}^{\prime}
$$

for some $i_{0}$. Therefore, by our strongly generic assumption, this implies that $n+1=$ $\operatorname{dim}(R)=\operatorname{dim}(T)=s$ and $f_{1}, \ldots, f_{s}$ indeed form a regular sequence.

\section{Symbolic POWERS of SATURATED UNIFORM $a$-FOLD PRODUCT IDEAL}

Inspired by the above work on the graded minimal free resolution of $I_{a}\left(f_{1}^{m_{1}} \cdots f_{s}^{m_{s}}\right)$, it is natural to seek a detailed description of the Betti table of $I_{a}\left(z_{1}^{m_{1}} \cdots z_{s}^{m_{s}}\right)$ in $T=$ $\mathbb{K}\left[z_{1}, \ldots, z_{s}\right]$. A starting point will be treating the uniform case when $m_{1}=\cdots=m_{s}=b$. This case can be handled with ease by the results from [3]. Indeed, more information can be retrieved. We will be able to scrutinize the projective dimension of this ideal, which will in turn allow us to describe its symbolic powers in some nice cases, via the decomposition work in Section 2. After that, we will study some asymptotic quantities related to the symbolic powers in these cases.

But first, we have to recall some notations from [3]. A sequence $\boldsymbol{\lambda}=\left(\lambda_{1}, \ldots, \lambda_{s}\right)$ of non-negative integers is called a partition of $d$ of length $s$, if $\lambda_{1} \leq \cdots \leq \lambda_{s}$ and $|\boldsymbol{\lambda}|:=$ $\lambda_{1}+\cdots+\lambda_{s}=d$. The symmetric group $\mathfrak{S}_{s}$ acts on $T=\mathbb{K}\left[z_{1}, \ldots, z_{s}\right]$ by permutations of the variables. Let $I \subset T$ be an $\mathfrak{S}_{s}$-fixed monomial ideal. Set

$$
\mathcal{P}(I):=\left\{\boldsymbol{\lambda} \text { is a partition of length } s: \boldsymbol{z}^{\boldsymbol{\lambda}} \in I\right\}
$$

and

$$
\Lambda(I):=\left\{\boldsymbol{\lambda}: \boldsymbol{z}^{\boldsymbol{\lambda}} \text { is a minimal monomial generator of } I\right\} .
$$

It is clear that $\Lambda(I)$ is the set of minimal elements in $\mathcal{P}(I)$ with respect to the partial ordering of componentwise comparison.

The ideal $I$ is called symmetric shifted if for every $\boldsymbol{\lambda}=\left(\lambda_{1}, \ldots, \lambda_{s}\right) \in \mathcal{P}(I)$ (or equivalently, $\boldsymbol{\lambda} \in \Lambda(I))$ and $1 \leq k<s$ with $\lambda_{k}<\lambda_{s}$, one has $\boldsymbol{z}^{\boldsymbol{\lambda}} z_{k} / z_{s} \in I$.

Given any partition $\boldsymbol{\lambda}=\left(\lambda_{1}, \ldots, \lambda_{s}\right)$ of $d$, one defines

$$
p(\boldsymbol{\lambda}):=\left|\left\{k: \lambda_{k}<\lambda_{s}-1\right\}\right| \text { and } r(\boldsymbol{\lambda}):=\left|\left\{k: \lambda_{k}=\lambda_{s}\right\}\right| .
$$

Besides, its truncation is $\boldsymbol{\lambda}_{\leq k}:=\left(\lambda_{1}, \ldots, \lambda_{k}\right)$, and its type is type $(\boldsymbol{\lambda}):=\left(t_{0}, \ldots, t_{d}\right)$ where $t_{i}=\left|\left\{k: \lambda_{k}=i\right\}\right|$. Furthermore, we set type $(\boldsymbol{\lambda}) !:=\prod_{i} t_{i} !$.

The key result that we shall apply repeatedly is the following. 
Lemma 4.1 ([3, Theorem 3.2, Corollary 5.6]). If $I \subset T$ is symmetric shifted, then $I$ has linear quotients and

$$
\beta_{i, i+d}(I)=\sum_{\boldsymbol{\lambda} \in \Lambda(I),|\boldsymbol{\lambda}|=d}\left(\sum_{k+l=i} \frac{p(\boldsymbol{\lambda}) !}{\operatorname{type}\left(\boldsymbol{\lambda}_{\leq p}(\boldsymbol{\lambda})\right) !}\left(\begin{array}{c}
s \\
p(\boldsymbol{\lambda})
\end{array}\right)\left(\begin{array}{c}
s-p(\boldsymbol{\lambda}) \\
r(\boldsymbol{\lambda})+k
\end{array}\right)\left(\begin{array}{c}
r(\boldsymbol{\lambda})+k-1 \\
k
\end{array}\right)\left(\begin{array}{c}
p(\boldsymbol{\lambda}) \\
l
\end{array}\right)\right) .
$$

Back to our uniform $a$-fold product ideal $I=I_{a}\left(z_{1}^{b} \cdots z_{s}^{b}\right)$ in $T=\mathbb{K}\left[z_{1}, \ldots, z_{s}\right]$. Then

$$
\Lambda(I)=\left\{\boldsymbol{\lambda}=\left(\lambda_{1}, \ldots, \lambda_{s}\right): \boldsymbol{\lambda} \text { is a partition of } a \text { with } \lambda_{s} \leq b\right\} .
$$

One can check with ease that $I$ is symmetric shifted. Consequently, its Betti table is clear by the previous lemma.

Example 4.2. Consider the ideal $I=I_{a}\left(z_{1}^{2} \cdots z_{s}^{2}\right)$ in $T=\mathbb{K}\left[z_{1}, \ldots, z_{s}\right]$. This ideal is symmetric shifted and $\Lambda(I)$ consists of partitions

$$
\boldsymbol{\lambda}_{u, v}:=\left(0^{s-u-v}, 1^{u}, 2^{v}\right)=(\underbrace{0, \ldots, 0}_{s-u-v}, \underbrace{1, \ldots, 1}_{u}, \underbrace{2, \ldots, 2}_{v})
$$

with $u+2 v=a$ and $v \geq 1$, as well as the partition

$$
\boldsymbol{\lambda}_{a, 0}:=\left(0^{s-a}, 1^{a}\right)=(\underbrace{0, \ldots, 0}_{s-a}, \underbrace{1, \ldots, 1}_{a}) .
$$

It is easy to see that

$$
p\left(\boldsymbol{\lambda}_{a, 0}\right)=0 \text { and } r\left(\boldsymbol{\lambda}_{a, 0}\right)=a .
$$

And if $v>0$, then

$$
p\left(\boldsymbol{\lambda}_{u, v}\right)=s-u-v \text { and } r\left(\boldsymbol{\lambda}_{u, v}\right)=v .
$$

Therefore, the formula (16) says

$$
\begin{aligned}
\beta_{i, i+a}(I) & =\sum_{k+l=i} \frac{0 !}{0 !}\left(\begin{array}{l}
s \\
0
\end{array}\right)\left(\begin{array}{c}
s-0 \\
a+k
\end{array}\right)\left(\begin{array}{c}
a+k-1 \\
k
\end{array}\right)\left(\begin{array}{l}
0 \\
l
\end{array}\right) \\
& +\sum_{v=1}^{\lfloor a / 2\rfloor} \sum_{k+l=i} \frac{(s+v-a) !}{(s+v-a) !}\left(\begin{array}{c}
s \\
s+v-a
\end{array}\right)\left(\begin{array}{c}
a-v \\
v+k
\end{array}\right)\left(\begin{array}{c}
v+k-1 \\
k
\end{array}\right)\left(\begin{array}{c}
s+v-a \\
l
\end{array}\right) \\
& =\left(\begin{array}{c}
s \\
a+i
\end{array}\right)\left(\begin{array}{c}
a+i-1 \\
i
\end{array}\right)+\sum_{v=1}^{\lfloor a / 2\rfloor} \sum_{k+l=i}\left(\begin{array}{c}
s \\
s+v-a
\end{array}\right)\left(\begin{array}{c}
a-v \\
v+k
\end{array}\right)\left(\begin{array}{c}
v+k-1 \\
k
\end{array}\right)\left(\begin{array}{c}
s+v-a \\
l
\end{array}\right) .
\end{aligned}
$$

For instance, when $a=3$ and $s=4$, the above formula says that

$$
\beta_{0,3}(I)=16, \quad \beta_{1,4}(I)=33, \quad \beta_{2,5}(I)=24, \quad \beta_{3,6}(I)=6, \quad \beta_{4,7}(I)=0 .
$$

It agrees with the following Betti table given by Macaulay2 [14]:

$$
\begin{array}{rrrrrr} 
& 0 & 1 & 2 & 3 & 4 \\
\text { total : } & 1 & 16 & 33 & 24 & 6 \\
0: & 1 & . & . & . & . \\
1: & . & . & . & . & . \\
2: & . & 16 & 33 & 24 & 6
\end{array}
$$


4.1. Projective dimension and primary-type decomposition. The ultimate aim of this subsection is to give a clean formula for the symbolic powers of the ideal of generalized star configuration $I_{a}\left(\mathcal{F}^{b}\right)=I_{a}\left(f_{1}^{b} \cdots f_{s}^{b}\right)$ in some nice cases. To achieve that, we start with investigating the projective dimension in the monomial case.

Proposition 4.3. Consider the uniform a-fold product ideal $I=I_{a}\left(z_{1}^{b} \cdots z_{s}^{b}\right)$ in $T=$ $\mathbb{K}\left[z_{1}, \ldots, z_{s}\right]$ with $b \leq a \leq b s$.

(i) The ideal $I$ is saturated if and only if $a>(b-1) s+1$.

(ii) If $a>(b-1) s+1$, then $\operatorname{proj}^{\operatorname{dim}_{T}}(T / I)=s-\delta$ for $\delta:=a-(b-1) s-1$.

Proof. Depending on the parameter $a$, we have two cases.

(a) If $b \leq a \leq s(b-1)+1$, we can always find some $\boldsymbol{\lambda}_{0}=\left(\lambda_{1}, \ldots, \lambda_{s}\right) \in \Lambda(I)$ such that $\lambda_{s-1}<\lambda_{s}=b$. This in particular means $r\left(\boldsymbol{\lambda}_{0}\right)=1$. Now, we consider the formula (16) with $i=s-1, \boldsymbol{\lambda}=\boldsymbol{\lambda}_{0}, k=s-p\left(\boldsymbol{\lambda}_{0}\right)-1$ and $l=p\left(\boldsymbol{\lambda}_{0}\right)$. This combination of setting contributes a positive value to the summation. Hence $\beta_{s-1, s-1+a}(I) \neq 0$ and $\operatorname{proj} \operatorname{dim}(T / I)=s=\operatorname{dim}(T)$. This implies that $\operatorname{depth}(T / I)=0$ and $I$ is not saturated.

(b) If $a>(b-1) s+1$, then for each fixed $\boldsymbol{\lambda}=\left(\lambda_{1}, \ldots, \lambda_{s}\right) \in \Lambda(I)$, we will have $\lambda_{s}=b$. As $|\boldsymbol{\lambda}|=a=(b-1) s+1+\delta, r(\boldsymbol{\lambda}) \geq 1+\delta$. Now for $i \geq s-\delta$,

$$
(r(\boldsymbol{\lambda})+k)+l=r(\boldsymbol{\lambda})+i \geq s+1>(s-p(\boldsymbol{\lambda}))+p(\boldsymbol{\lambda})=s,
$$

giving

$$
\left(\begin{array}{c}
s-p(\boldsymbol{\lambda}) \\
r(\boldsymbol{\lambda})+k
\end{array}\right)\left(\begin{array}{c}
p(\boldsymbol{\lambda}) \\
l
\end{array}\right)=0
$$

in the formula (16). This implies that the total Betti number $\beta_{i}(I)=0$ for $i \geq s-\delta$.

Meanwhile, we can find the partition $\boldsymbol{\lambda}_{0}=\left((b-1)^{s-1-\delta}, b^{1+\delta}\right) \in \Lambda(I)$ with $r\left(\boldsymbol{\lambda}_{0}\right)=$ $\delta+1$. An argument as above will show that $\beta_{s-\delta-1}(I) \neq 0$. Therefore, $\operatorname{proj} \operatorname{dim}(I)=$ $s-\delta-1$, or equivalently, $\operatorname{proj} \operatorname{dim}(T / I)=s-\delta$. Whence, $I$ is saturated.

Proposition 4.4. With the assumptions in Setting 2.8, we further assume that $a>(b-$ $1) s+1$ and write $\delta:=a-(b-1) s-1$. If $\delta \geq s-n$ and $\mathcal{F}=\left\{f_{1}, \ldots, f_{s}\right\}$ is a set of $(s-\delta)$-generic $d$-forms in $R=\mathbb{K}\left[x_{0}, \ldots, x_{n}\right]$, then

$$
I_{a}\left(\mathcal{F}^{b}\right)=\bigcap_{c=c_{0}}^{s-\delta}\left(\bigcap_{1 \leq i_{1}<\cdots<i_{c} \leq s}\left\langle f_{i_{1}}, \ldots, f_{i_{c}}\right\rangle^{\mu_{a}^{0}+b\left(c-c_{0}\right)}\right) .
$$

Proof. Consider as well $\mathcal{F}_{\boldsymbol{z}}=\left\{z_{1}, \ldots, z_{s}\right\}$ in $T=\mathbb{K}\left[z_{1}, \ldots, z_{s}\right]$. We have seen in the proof of Theorem 3.7 that $I_{a}\left(\mathcal{F}_{\boldsymbol{z}}^{b}\right)$ has linear quotients. It follows from Corollary 3.11 and Proposition 4.3 that

$$
\operatorname{proj} \operatorname{dim}_{R}\left(R / I_{a}\left(\mathcal{F}^{b}\right)\right) \leq \operatorname{proj} \operatorname{dim}_{T}\left(T / I_{a}\left(\mathcal{F}_{\boldsymbol{z}}^{b}\right)\right)=s-\delta \leq n .
$$

Therefore, we can apply the fact in Remark 2.6 and obtain the decomposition in Theorem 2.5 with $\hat{c}=s-\delta$.

In that decomposition, the exponent $\mu_{a}\left(i_{1}, \ldots, i_{c}\right)=\mu_{a}^{0}+b\left(c-c_{0}\right)$ for $c_{0} \leq c \leq s-\delta$ by the direct computation at the end of Section 2. And when $c<c_{0}$, the exponent $\mu_{a}\left(i_{1}, \ldots, i_{c}\right)<$ 0 . Obviously, we can remove these redundant components from the decomposition. 
Remark 4.5. In order to study the symbolic powers of $I_{a}\left(\mathcal{F}^{b}\right)$ later, we still need to determine its associate primes via Proposition 4.4. By the decomposition formula there, it is clear that $\operatorname{Ass}\left(R / I_{a}\left(\mathcal{F}^{b}\right)\right)$ is a subset of the disjoint union

$$
\mathcal{A}:=\bigsqcup_{\substack{c_{0} \leq c \leq s-\delta \\ 1 \leq i_{1}<\cdots<i_{c} \leq s}} \operatorname{Ass}\left(R /\left\langle f_{i_{1}}, \ldots, f_{i_{c}}\right\rangle\right),
$$

since the forms in $\mathcal{F}$ are locally complete intersections. On the other hand, let us take arbitrary $\mathfrak{p} \in \mathcal{A}$. Say, $\mathfrak{p} \in \operatorname{Ass}\left(R /\left\langle f_{1}, \ldots, f_{c}\right\rangle\right)$. Then $I_{a}\left(\mathcal{F}^{b}\right) R_{\mathfrak{p}}=I_{a-b(s-c)}\left(f_{1}^{b} \cdots f_{c}^{b}\right) R_{\mathfrak{p}}$ by our previous calculation in the equation (6). Notice that $\operatorname{dim}\left(R_{\mathfrak{p}}\right)=c$ and the images of $f_{1}, \ldots, f_{c}$ in $R_{\mathfrak{p}}$ form a regular sequence. Since

$$
a-b(s-c) \leq(b-1) c+1
$$

for $c_{0} \leq c \leq s-\delta$, it follows from Proposition 4.3 that $I_{a-b(s-c)}\left(f_{1}^{b} \cdots f_{c}^{b}\right) R_{\mathfrak{p}}$ is not saturated in $R_{\mathfrak{p}}$. Therefore, $\mathfrak{p} R_{\mathfrak{p}}$ is an associated prime of $R_{\mathfrak{p}} / I_{a-b(s-c)}\left(f_{1}^{b} \cdots f_{c}^{b}\right) R_{\mathfrak{p}}$ and equivalently $\mathfrak{p}$ is an associated prime of $R / I_{a}\left(\mathcal{F}^{b}\right)$. In short, $\mathcal{A}=\operatorname{Ass}\left(R / I_{a}\left(\mathcal{F}^{b}\right)\right)$.

Consequently, the decomposition in Proposition 4.4 is irredundant in the obvious sense.

Now, we are ready to state the first main result of this section.

Theorem 4.6. With the assumptions in Proposition 4.4, the symbolic powers of $I_{a}\left(\mathcal{F}^{b}\right)$ can be written as

$$
\begin{aligned}
I_{a}\left(\mathcal{F}^{b}\right)^{(m)} & =\bigcap_{c=c_{0}}^{s-\delta}\left(\bigcap_{1 \leq i_{1}<\cdots<i_{c} \leq s}\left\langle f_{i_{1}}, \ldots, f_{i_{c}}\right\rangle^{m\left(\mu_{a}^{0}+b\left(c-c_{0}\right)\right)}\right) \\
& =\bigcap_{c=c_{0}}^{s-\delta} I_{c, \mathcal{F}}^{\left(m\left(\mu_{a}^{0}+b\left(c-c_{0}\right)\right)\right)} .
\end{aligned}
$$

Proof. We will adopt the symbol $\mathcal{A}$ in the Remark 4.5. By definition,

$$
I_{a}\left(\mathcal{F}^{b}\right)^{(m)}=\bigcap_{\mathfrak{p} \in \mathcal{A}}\left(I_{a}\left(\mathcal{F}^{b}\right)^{m} R_{\mathfrak{p}} \cap R\right) .
$$

For each $\mathfrak{p} \in \mathcal{A}$ with $h t(\mathfrak{p})=c$, we can find, for instance, $f_{1}, \ldots, f_{c} \in \mathfrak{p}$ while $f_{c+1}, \ldots, f_{s} \notin$ $\mathfrak{p}$. Whence,

$$
\begin{aligned}
I_{a}\left(\mathcal{F}^{b}\right)^{m} R_{\mathfrak{p}} & =I_{a-b(s-c)}\left(f_{1}^{b} \cdots f_{c}^{b}\right)^{m} R_{\mathfrak{p}} \\
& =I_{m a-m b(s-c)}\left(f_{1}^{m b} \cdots f_{c}^{m b}\right) R_{\mathfrak{p}} \\
& =\bigcap_{c_{0} \leq c^{\prime} \leq c}\left(\bigcap_{1 \leq i_{1}<\cdots<i_{c^{\prime}} \leq c}\left\langle f_{i_{1}}, \ldots, f_{i_{c^{\prime}}}\right\rangle^{m\left(\mu_{a}^{0}+b\left(c^{\prime}-c_{0}\right)\right)} R_{\mathfrak{p}}\right) \\
& =\bigcap_{c_{0} \leq c^{\prime} \leq s-\delta}\left(\bigcap_{1 \leq i_{1}<\cdots<i_{c^{\prime}} \leq s}\left\langle f_{i_{1}}, \ldots, f_{i_{c^{\prime}}}\right\rangle^{m\left(\mu_{a}^{0}+b\left(c^{\prime}-c_{0}\right)\right)} R_{\mathfrak{p}}\right) .
\end{aligned}
$$

The first three equalities are due to formulas (6) , (9) and Lemma 2.2 respectively. The last one is also clear, since any $f_{j}$ not in $\mathfrak{p}$ will lead to the localization ideal being $R_{\mathfrak{p}}$. And 
this induces

$$
I_{a}\left(\mathcal{F}^{b}\right)^{(m)}=\bigcap_{\mathfrak{p} \in \mathcal{A}}\left(\bigcap_{c=c_{0}}^{s-\delta}\left(\bigcap_{1 \leq i_{1}<\cdots<i_{c} \leq s}\left\langle f_{i_{1}}, \ldots, f_{i_{c}}\right\rangle^{m\left(\mu_{a}^{0}+b\left(c-c_{0}\right)\right)} R_{\mathfrak{p}} \cap R\right)\right) .
$$

At the same time, for each $c$ with $c_{0} \leq c \leq s-\delta$, we have

$$
\begin{aligned}
I_{c, \mathcal{F}}^{\left(m\left(\mu_{a}^{0}+b\left(c-c_{0}\right)\right)\right)} & =\bigcap_{1 \leq i_{1}<\cdots<i_{c} \leq s}\left\langle f_{i_{1}}, \ldots, f_{i_{c}}\right\rangle^{m\left(\mu_{a}^{0}+b\left(c-c_{0}\right)\right)} \\
& \subseteq \bigcap_{\mathfrak{p} \in \mathcal{A}}\left(\bigcap_{1 \leq i_{1}<\cdots<i_{c} \leq s}\left\langle f_{i_{1}}, \ldots, f_{i_{c}}\right\rangle^{m\left(\mu_{a}^{0}+b\left(c-c_{0}\right)\right)} R_{\mathfrak{p}} \cap R\right) \\
& \subseteq \bigcap_{\substack{\mathfrak{p} \in \mathcal{A} \\
\operatorname{ht}(\mathfrak{p})=c}}\left(\bigcap_{1 \leq i_{1}<\cdots<i_{c} \leq s}\left\langle f_{i_{1}}, \ldots, f_{i_{c}}\right\rangle^{m\left(\mu_{a}^{0}+b\left(c-c_{0}\right)\right)} R_{\mathfrak{p}} \cap R\right) \\
& =I_{c, \mathcal{F}}^{\left(m\left(\mu_{a}^{0}+b\left(c-c_{0}\right)\right)\right)} .
\end{aligned}
$$

Here, we need [13, Theorem 3.6(1)] for the two equalities.

After putting together every piece of information, we arrive at the expected formulas.

4.2. Monomial case. To study the symbolic powers of the uniform $a$-fold product ideal $I_{a}\left(\mathcal{F}^{b}\right)$, we have to check with its monomial prototype $I_{a}\left(z_{1}^{b} \cdots z_{s}^{b}\right) \subset T=\mathbb{K}\left[z_{1}, \ldots, z_{s}\right]$ first. So, temporarily, we shift our focus to the monomial case. As the initial step, we can strengthen the last piece of Proposition 4.3 as follows.

Proposition 4.7. With the assumptions in Setting[2.8, we consider the set $\mathcal{F}_{\boldsymbol{z}}=\left\{z_{1}, \ldots, z_{s}\right\}$ in the polynomial ring $\mathbb{K}\left[z_{1}, \ldots, z_{s}\right]$. Suppose that $(b-1) s+1<a \leq b s$ and we write $\delta:=a-(b-1) s-1$. Then, for each positive integer $m, \operatorname{proj}^{\operatorname{dim}_{T}}\left(T / I_{a}\left(\mathcal{F}_{z}^{b}\right)^{(m)}\right)=s-\delta$ and $\operatorname{reg}_{T}\left(T / I_{a}\left(\mathcal{F}_{\boldsymbol{z}}^{b}\right)^{(m)}\right)=m\left(\mu_{a}^{0}+b\left(s-c_{0}\right)\right)-1$.

Proof. Firstly, in order to apply Lemma 4.1, we need to verify that $I=I_{a}\left(\mathcal{F}_{\boldsymbol{z}}^{b}\right)^{(m)}$ is symmetric shifted. Notice that this ideal is obviously $\mathfrak{S}_{s}$-invariant. Thus, we will take arbitrary partition $\boldsymbol{\lambda}=\left(\lambda_{1}, \ldots, \lambda_{s}\right) \in \mathcal{P}\left(I_{a}\left(\mathcal{F}_{z}^{b}\right)^{(m)}\right)$. It follows from Theorem 4.6 that this is equivalent to saying that $\boldsymbol{z}^{\boldsymbol{\lambda}} \in I_{c}:=I_{c, \mathcal{F}_{z}}^{\left(m\left(\mu_{a}^{0}+b\left(c-c_{0}\right)\right)\right)}$ for each $c=c_{0}, c_{0}+1, \ldots, s-\delta$. Notice that $I_{c}$ is symmetric shifted by [3, Theorem 4.3] for each $c$. Therefore, if $\lambda_{k}<\lambda_{s}$, then $\boldsymbol{z}^{\boldsymbol{\lambda}} z_{k} / z_{s} \in I_{c}$ for each such $c$. Consequently, $\boldsymbol{z}^{\boldsymbol{\lambda}} z_{k} / z_{s} \in I=\bigcap_{c} I_{c}$, meaning $I$ is symmetric shifted.

Furthermore, by the above argument, a partition $\boldsymbol{\lambda} \in \mathcal{P}\left(I_{a}\left(\mathcal{F}_{z}^{b}\right)^{(m)}\right)$ if and only if $\boldsymbol{z}^{\boldsymbol{\lambda}} \in I_{c}$ for $c_{0} \leq c \leq s-\delta$, and by [3], Proposition 4.1], if and only if $\left|\boldsymbol{\lambda}_{\leq c}\right| \geq m\left(\mu_{a}^{0}+b\left(c-c_{0}\right)\right)$ for every such $c$. Thus, each $\boldsymbol{\lambda} \in \Lambda\left(I_{a}\left(\mathcal{F}_{\boldsymbol{z}}^{b}\right)^{(m)}\right)$ satisfies $\lambda_{s-\delta}=\cdots=\lambda_{s}$. In particu-

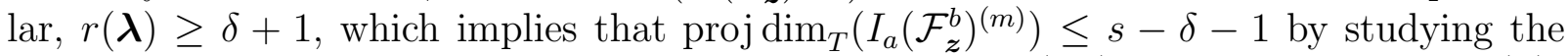
formula (16). Meanwhile, we do find $\boldsymbol{\lambda}_{0}=\left((m(b-1))^{s-(\delta+1)},(m b)^{\delta+1}\right) \in \Lambda\left(I_{a}\left(\mathcal{F}_{z}^{b}\right)^{(m)}\right)$ with $r\left(\boldsymbol{\lambda}_{0}\right)=\delta+1$. This means that proj $\operatorname{dim}_{T}\left(I_{a}\left(\mathcal{F}_{z}^{b}\right)^{(m)}\right)=s-\delta-1$, and equivalently $\operatorname{proj} \operatorname{dim}_{T}\left(T / I_{a}\left(\mathcal{F}_{\boldsymbol{z}}^{b}\right)^{(m)}\right)=s-\delta$.

To determine the Castelnuovo-Mumford regularity, it suffices to find

$$
\max \left\{|\boldsymbol{\lambda}|: \boldsymbol{\lambda} \in \Lambda\left(I_{a}\left(\mathcal{F}_{\boldsymbol{z}}^{b}\right)^{(m)}\right)\right\}
$$

by Lemma 4.1. Since $c_{0} \leq s-\delta$, we have two cases. 
(a) Suppose that $c_{0}=s-\delta$. It is clear that $\boldsymbol{\lambda} \in \Lambda\left(I_{a}\left(\mathcal{F}_{\boldsymbol{z}}^{b}\right)^{(m)}\right)$ if and only if $\left|\boldsymbol{\lambda}_{\leq c_{0}}\right|=$ $m \mu_{a}^{0}$ with $\lambda_{c_{0}}=\cdots=\lambda_{s}$. Thus, the maximum is $m \mu_{a}^{0}\left(s-c_{0}+1\right)$, achieved at $\left(0^{c_{0}-1},\left(m \mu_{a}^{0}\right)^{s-c_{0}+1}\right)$.

(b) Suppose that $c_{0}<s-\delta$ and take arbitrary $\boldsymbol{\lambda} \in \Lambda\left(I_{a}\left(\mathcal{F}_{\boldsymbol{z}}^{b}\right)^{(m)}\right)$.

We claim first that $\lambda_{s-\delta} \leq m b$. Suppose for contradiction that $\lambda_{s-\delta}>m b$. Let $t_{0}:=\min \left\{c_{0} \leq t \leq s-\delta: \lambda_{t}>m b\right\}$. If $t_{0}=c_{0}$, then $\boldsymbol{\lambda}^{\prime}=\left(0^{c_{0}-1},(m b)^{s-c_{0}+1}\right) \in$ $\mathcal{P}\left(I_{a}\left(\mathcal{F}_{\boldsymbol{z}}^{b}\right)^{(m)}\right.$. If $t_{0}>c_{0}$, then $\left|\boldsymbol{\lambda}_{\leq t_{0}-1}\right| \geq m\left(\mu_{a}^{0}+b\left(t_{0}-1-c_{0}\right)\right)$. As $\lambda_{c}>m b$ for $c \geq t_{0}$, we also have $\left|\boldsymbol{\lambda}_{\leq c}^{\prime \prime}\right| \geq m\left(\mu_{a}^{0}+b\left(c-c_{0}\right)\right)$ for $c \geq t_{0}$ and $\boldsymbol{\lambda}^{\prime \prime}=\left(\lambda_{1}, \ldots, \lambda_{c-1}, m b, \ldots, m b\right)$. This implies that $\boldsymbol{\lambda}^{\prime \prime} \in \mathcal{P}\left(I_{a}\left(\mathcal{F}_{\boldsymbol{z}}^{b}\right)^{(m)}\right)$. In both subcases, we have a contradiction to the minimality of $\boldsymbol{\lambda}$. Thus, $\lambda_{s-\delta} \leq m b$.

We next claim that $\left|\boldsymbol{\lambda}_{\leq s-\delta}\right|=m\left(\mu_{a}^{0}+b\left(s-\delta-c_{0}\right)\right)$ in this situation. Suppose that this is not true. It follows that $\left|\boldsymbol{\lambda}_{\leq s-\delta}\right|>m\left(\mu_{a}^{0}+b\left(s-\delta-c_{0}\right)\right)$. As $\mu_{a}^{0} \leq b$, we will additionally have $\left|\boldsymbol{\lambda}_{\leq t}\right|>m\left(\mu_{a}^{0}+\vec{b}\left(t-c_{0}\right)\right)$ for $c_{0} \leq t \leq s-\delta$. Now, suppose that $t_{1}=\min \left\{1 \leq t \leq s: \lambda_{t}>0\right\}$. If we take $\boldsymbol{\lambda}^{\prime \prime \prime}=\left(0, \ldots, 0, \lambda_{t_{1}}-1, \lambda_{t_{1}+1}, \ldots, \lambda_{s}\right)$, then $\left|\boldsymbol{\lambda}_{\leq t}^{\prime \prime \prime}\right| \geq m\left(\mu_{a}^{0}+b\left(t-c_{0}\right)\right)$ for $c_{0} \leq t \leq s-\delta$. This implies that $\boldsymbol{\lambda}^{\prime \prime \prime} \in \mathcal{P}\left(I_{a}\left(\mathcal{F}_{\boldsymbol{z}}^{b}\right)^{(m)}\right)$, contradicting the minimality of $\boldsymbol{\lambda}$.

Now, as $\left|\boldsymbol{\lambda}_{\leq s-\delta}\right|=m\left(\mu_{a}^{0}+b\left(s-\delta-c_{0}\right)\right)$ and $\lambda_{s-\delta}=\cdots=\lambda_{s} \leq m b$, the maximum of $|\boldsymbol{\lambda}|$ is $m\left(\mu_{a}^{0}+b\left(s-c_{0}\right)\right)$, achieved at $\left(0^{c_{0}-1}, m \mu_{a}^{0},(m b)^{s-c_{0}}\right)$.

Notice that $c_{0}=s-\delta$ precisely when $s-\delta=\left\lceil\frac{s-\delta}{b}\right\rceil$, and precisely when $b=1$ or $\delta=s-1$. Whence, the two maxima computed above all agree with ma. Therefore, we can simply take the second format and obtain $\operatorname{reg}_{T}\left(I_{a}\left(\mathcal{F}_{\boldsymbol{z}}^{b}\right)^{(m)}\right)=m\left(\mu_{a}^{0}+b\left(s-c_{0}\right)\right)$ by Lemma 4.1.

Next, we are going to study the resurgence of the generalized star configuration in the monomial case. Recall that if $I$ is a nonzero graded ideal in the standard graded $\operatorname{ring} R$, the resurgence of $I$ is defined to be

$$
\rho(I):=\sup \left\{\frac{m}{r}: I^{(m)} \nsubseteq I^{r}\right\} .
$$

We will always denote by $\alpha(I)$ the least degree of nonzero forms in $I$. Meanwhile, the Waldschmidt constant $\widehat{\alpha}(I)$ of $I$ is defined to be

$$
\widehat{\alpha}(I):=\lim _{m \rightarrow \infty} \frac{\alpha\left(I^{(m)}\right)}{m} .
$$

This limit is known to exist and satisfies

$$
\frac{\alpha(I)}{\widehat{\alpha}(I)} \leq \rho(I)
$$

by [5. Lemma 2.3.1 and Theorem 1.2.1].

Proposition 4.8. With the assumptions in Proposition 4.7, for the uniform a-fold product ideal $I=I_{a}\left(\mathcal{F}_{\boldsymbol{z}}^{b}\right)$, we have

$$
\rho(I)=\frac{\alpha(I)}{\widehat{\alpha}(I)}=\frac{a(s-\delta)}{s\left(\mu_{a}^{0}+b\left(s-\delta-c_{0}\right)\right)} .
$$

Proof. Partitions $\boldsymbol{\lambda} \in \mathcal{P}\left(I_{a}\left(\mathcal{F}_{\boldsymbol{z}}^{b}\right)^{(m)}\right)$ are characterized by the requirements

$$
\left|\boldsymbol{\lambda}_{\leq c}\right| \geq m\left(\mu_{a}^{0}+b\left(c-c_{0}\right)\right) \text { for } c_{0} \leq c \leq s-\delta .
$$

It is clear that $\lambda_{c} \geq\left\lceil\frac{m\left(\mu_{a}^{0}+b\left(c-c_{0}\right)\right)}{c}\right\rceil$ for each such $c$. Furthermore, since $\mu_{a}^{0} \leq b$ and $c_{0} \leq s-\delta$, one has $\frac{m \mu_{a}^{0}}{c_{0}} \leq \frac{m\left(\mu_{a}^{0}+b\left(s-\delta-c_{0}\right)\right)}{c_{0}+\left(s-\delta-c_{0}\right)}$ and consequently $\left\lceil\frac{m \mu_{a}^{0}}{c_{0}}\right\rceil \leq\left\lceil\frac{m\left(\mu_{a}^{0}+b\left(s-\delta-c_{0}\right)\right)}{s-\delta}\right\rceil$. 
Meanwhile, $\boldsymbol{\lambda} \in \mathcal{P}\left(I_{a}\left(\mathcal{F}_{\boldsymbol{z}}^{b}\right)^{r}\right)=\mathcal{P}\left(I_{a r}\left(\mathcal{F}_{\boldsymbol{z}}^{b r}\right)\right)$ if and only if $\sum_{i} \min \left(\lambda_{i}, b r\right) \geq a r$. Now, for the containment $I_{a}\left(\mathcal{F}_{\boldsymbol{z}}^{b}\right)^{(m)} \subseteq I_{a}\left(\mathcal{F}_{\boldsymbol{z}}^{b}\right)^{r}$ with $m \geq r \geq 1$, we have three cases.

(a) Suppose that $\lambda_{c_{0}} \geq b r$. Then, $\lambda_{i} \geq b r$ for $c_{0} \leq i \leq s$. Whence, the minimum of $\sum_{i=1}^{s} \min \left(\lambda_{i}, b r\right)$ in this case is exactly $\operatorname{br}\left(s-c_{0}+1\right)$. Now, the requirement for the containment is

$$
\operatorname{br}\left(s-c_{0}+1\right) \geq a r,
$$

which holds automatically by (10) and (11). This means that the requirement for the containment is void in this case.

(b) Suppose that $\lambda_{s-\delta}<b r$. Then

$$
\min \left(\lambda_{i}, b r\right) \geq \lambda_{s-\delta} \geq\left\lceil\frac{m\left(\mu_{a}^{0}+b\left(s-\delta-c_{0}\right)\right)}{s-\delta}\right\rceil
$$

for $s-\delta+1 \leq i \leq s$. Therefore, the minimum of $\sum_{i=1}^{s} \min \left(\lambda_{i}, b r\right)$ in this case coincides with

$$
A:=m\left(\mu_{a}^{0}+b\left(s-\delta-c_{0}\right)\right)+\delta\left\lceil\frac{m\left(\mu_{a}^{0}+b\left(s-\delta-c_{0}\right)\right)}{s-\delta}\right\rceil .
$$

Whence, the condition for the containment is simply $A \geq a r$. If we write

$$
m\left(\mu_{a}^{0}+b\left(s-\delta-c_{0}\right)\right)=q_{0}(s-\delta)+q_{1} \quad \text { with } 1 \leq q_{1} \leq s-\delta,
$$

then the requirement $A \geq a r$ is equivalent to saying

$$
r \leq \frac{q_{0} s+q_{1}+\delta}{a}
$$

(c) Suppose that $\lambda_{c_{0}}<b r \leq \lambda_{s-\delta}$. Then let $c$ be the smallest such that $\lambda_{c} \geq b r$. It is clear that $c_{0}<c \leq s-\delta$. Similar to the above discussions, we find the minimum of $\sum_{i=1}^{s} \min \left(\lambda_{i}, b r\right)$ in this case agreeing with

$$
B:=m\left(\mu_{a}^{0}+b\left(c-c_{0}-1\right)\right)+(s-c+1) b r .
$$

Thus, the condition for the containment is simply $B \geq a r$, or equivalently $r \leq m$ by (10) and (11). Since $r \leq m$ is always true for $I_{a}\left(\mathcal{F}_{\boldsymbol{z}}^{b}\right)^{(m)} \subseteq I_{a}\left(\mathcal{F}_{\boldsymbol{z}}^{b}\right)^{r}$, the requirement for the containment is void in this case.

To sum up, $I_{a}\left(\mathcal{F}_{\boldsymbol{z}}^{b}\right)^{(m)} \nsubseteq I_{a}\left(\mathcal{F}_{\boldsymbol{z}}^{b}\right)^{r}$ if and only if

$$
\min \left\{\lambda_{s-\delta}: \boldsymbol{\lambda} \in \mathcal{P}\left(I_{a}\left(\mathcal{F}_{\boldsymbol{z}}^{b}\right)^{(m)}\right)\right\}=\left\lceil\frac{m\left(\mu_{a}^{0}+b\left(s-\delta-c_{0}\right)\right)}{s-\delta}\right\rceil<b r
$$

while $r>\frac{q_{0} s+q_{1}+\delta}{a}$. Whence,

$$
\frac{m}{r}<\frac{m a}{q_{0} s+q_{1}+\delta} \leq \frac{a(s-\delta)}{s\left(\mu_{a}^{0}+b\left(s-\delta-c_{0}\right)\right)} .
$$

The second inequality can be verified directly by paying attention to the assumptions in (17). Consequently, we have established

$$
\rho(I) \leq \frac{a(s-\delta)}{s\left(\mu_{a}^{0}+b\left(s-\delta-c_{0}\right)\right)} .
$$


On the other hand, it is clear that $\alpha(I)=a$, while

$$
\begin{aligned}
\alpha\left(I^{(m)}\right) & \geq \max \left\{\alpha\left(I_{c, \mathcal{F}_{\boldsymbol{z}}}^{\left(m\left(\mu_{a}^{0}+b\left(c-c_{0}\right)\right)\right)}\right): c_{0} \leq c \leq s-\delta\right\} \\
& \geq \alpha\left(I_{s-\delta, \mathcal{F}_{\boldsymbol{z}}}^{\left(m\left(\mu_{0}^{0}+b\left(s-\delta-c_{0}\right)\right)\right)}\right) \\
& =m\left(\mu_{a}^{0}+b\left(s-\delta-c_{0}\right)\right)+\left\lceil\frac{m\left(\mu_{a}^{0}+b\left(s-\delta-c_{0}\right)\right)}{s-\delta}\right\rceil \delta
\end{aligned}
$$

by Theorem 4.6 and [3, Proposition 4.1]. Notice that we can find a (unique) partition

$$
\boldsymbol{\lambda}_{0}=\left(\lambda_{1}, \ldots, \lambda_{s}\right) \in \Lambda\left(I_{s-\delta, \mathcal{F}_{\boldsymbol{z}}}^{\left(m\left(\mu_{a}^{0}+b\left(s-\delta-c_{0}\right)\right)\right)}\right)
$$

with

$$
\lambda_{1} \leq \cdots \leq \lambda_{s}=\left\lceil\frac{m\left(\mu_{a}^{0}+b\left(s-\delta-c_{0}\right)\right)}{s-\delta}\right\rceil \leq \lambda_{1}+1 .
$$

Obviously we have $\boldsymbol{\lambda}_{0} \in \mathcal{P}\left(I_{c, \mathcal{F}_{\boldsymbol{z}}}^{\left(m\left(\mu_{a}^{0}+b\left(c-c_{0}\right)\right)\right)}\right)$ for $c_{0} \leq c \leq s-\delta$. The existence of such $\boldsymbol{\lambda}_{0}$ implies that the comparisons in (18) and (19) are indeed equalities. Therefore,

$$
\begin{aligned}
\rho(I) & \geq \frac{\alpha(I)}{\widehat{\alpha}(I)}=a / \lim _{m \rightarrow \infty} \frac{m\left(\mu_{a}^{0}+b\left(s-\delta-c_{0}\right)\right)+\left\lceil\frac{m\left(\mu_{a}^{0}+b\left(s-\delta-c_{0}\right)\right)}{s-\delta}\right\rceil \delta}{m} \\
& =\frac{a(s-\delta)}{s\left(\mu_{a}^{0}+b\left(s-\delta-c_{0}\right)\right)} .
\end{aligned}
$$

And this completes the proof.

Here is some information regarding the symbolic defect in the monomial case.

Proposition 4.9. With the assumptions in Proposition 4.7, for the uniform a-fold product ideal $I=I_{a}\left(\mathcal{F}_{\boldsymbol{z}}^{b}\right)$ and the positive integer $m$, we assign

$$
\Lambda(I, m):=\left\{\boldsymbol{\lambda}:\left|\boldsymbol{\lambda}_{\leq s-\delta}\right|=m\left(\mu_{a}^{0}+b\left(s-\delta-c_{0}\right)\right) \text { and } \lambda_{s-\delta}=\lambda_{s-\delta+1}=\cdots=\lambda_{s}<m b\right\} .
$$

Now, the symbolic defect $\operatorname{sdefect}(I, m)$ is given by $\sum_{\boldsymbol{\lambda} \in \Lambda(I, m)} \frac{s !}{\operatorname{type}(\boldsymbol{\lambda}) !}$.

Proof. By reading the previous proof with $r=m$, we acknowledge that any partition $\boldsymbol{\lambda} \in \Lambda\left(I_{a}\left(\mathcal{F}_{\boldsymbol{z}}^{b}\right)^{(m)}\right)$ with $\boldsymbol{z}^{\boldsymbol{\lambda}} \notin I_{a}\left(\mathcal{F}_{\boldsymbol{z}}^{b}\right)^{m}$ satisfies the requirements that

$$
\left|\boldsymbol{\lambda}_{\leq c}\right| \geq m\left(\mu_{a}^{0}+b\left(c-c_{0}\right)\right)
$$

for all $c_{0} \leq c \leq s-\delta$, and

$$
\lambda_{s-\delta}=\lambda_{s-\delta+1}=\cdots=\lambda_{s}<m b .
$$

Since $\lambda_{c} \leq \lambda_{s-\delta} \leq m b-1$ for $c_{0} \leq c \leq s-\delta$. It follows that

$$
\begin{aligned}
\left|\boldsymbol{\lambda}_{\leq c}\right|=\left|\boldsymbol{\lambda}_{\leq s-\delta}\right|-\sum_{k=c}^{s-\delta-1} \lambda_{k} & \geq m\left(\mu_{a}^{0}+b\left(s-\delta-c_{0}\right)\right)-(s-\delta-c)(m b-1) \\
& >m\left(\mu_{a}^{0}+b\left(c-c_{0}\right)\right)
\end{aligned}
$$

when $c_{0} \leq c<s-\delta$. Consequently, it suffices to require (20) for $c=s-\delta$ solely.

Now, the multigraded module $I_{a}\left(\mathcal{F}_{\boldsymbol{z}}^{b}\right)^{(m)} / I_{a}\left(\mathcal{F}_{\boldsymbol{z}}^{b}\right)^{m}$ is minimally generated by the images of $\boldsymbol{z}^{\boldsymbol{\lambda}}$ where the partition $\boldsymbol{\lambda}$ satisfies

$$
\left|\boldsymbol{\lambda}_{\leq s-\delta}\right|=m\left(\mu_{a}^{0}+b\left(s-\delta-c_{0}\right)\right) \quad \text { and } \quad \lambda_{s-\delta}=\lambda_{s-\delta+1}=\cdots=\lambda_{s}<m b .
$$


We collect these partitions into the set $\Lambda(I, m)$.

The final piece of the proof is the well-known fact that $\left|\mathfrak{S}_{n} \cdot \boldsymbol{z}^{\boldsymbol{\lambda}}\right|=\frac{s !}{\operatorname{type}(\boldsymbol{\lambda}) !}$.

Example 4.10. Consider the ideal $I=I_{7}\left(z_{1}^{2} \cdots z_{5}^{2}\right)$ in $T=\mathbb{K}\left[z_{1}, \ldots, z_{5}\right]$. Then $\delta=1$, $c_{0}=2$ and $\mu_{a}^{0}=1$. Whence,

$$
\Lambda(I, 2)=\left\{\boldsymbol{\lambda}:\left|\boldsymbol{\lambda}_{\leq 4}\right|=10 \text { and } \lambda_{4}<4\right\}=\{(2,2,3,3,3),(1,3,3,3,3)\} .
$$

And consequently, $\operatorname{sdefect}(I, 2)=\frac{5 !}{2 ! 3 !}+\frac{5 !}{1 ! 4 !}=15$, agreeing with the computation by Macaulay2 [14] via the SymbolicPowers package.

4.3. General case. It is time to shift back our focus to symbolic powers of generalized star configurations of generic forms.

Proposition 4.11. With the assumptions in Proposition 4.4, let $\varphi: T=\mathbb{K}\left[z_{1}, \ldots, z_{s}\right] \rightarrow R$ be the homomorphism induced by $z_{i} \mapsto f_{i}$ for each $i$. For each fixed positive integer $m$, suppose that $\boldsymbol{F}_{\boldsymbol{0}}$ is a graded minimal free resolution of $T / I_{a}\left(\mathcal{F}_{\boldsymbol{z}}^{b}\right)^{(m)}$ for $\mathcal{F}_{\boldsymbol{z}}=\left\{z_{1}, \ldots, z_{s}\right\} \subseteq$ T. Then, $I_{a}\left(\mathcal{F}^{b}\right)^{(m)}=\varphi\left(I_{a}\left(\mathcal{F}_{\boldsymbol{z}}^{b}\right)^{(m)}\right)$ and $\boldsymbol{F}_{\bullet} \otimes_{T} R$ is a graded minimal free resolution of $R / I_{a}\left(\mathcal{F}^{b}\right)^{(m)}$. In particular, a minimal generating set of $I_{a}\left(\mathcal{F}^{b}\right)^{(m)}$ is given by

$$
\left\{\varphi(g) \mid g \in G\left(I_{a}\left(\mathcal{F}_{\boldsymbol{z}}^{b}\right)^{(m)}\right)\right\} .
$$

Here, $G\left(I_{a}\left(\mathcal{F}_{\boldsymbol{z}}^{b}\right)^{(m)}\right)$ is the minimal monomial generating set of the corresponding monomial ideal.

Proof. We prove by an induction on $s-\hat{c}$ where $\hat{c}:=\max \{c: \mathcal{F}$ is $c$-generic $\}$. Obviously, $s-\delta \leq \hat{c} \leq s-1$. If $s-\hat{c}=1, \mathcal{F}$ forms a regular sequence. Whence, $\varphi$ is flat and the claim is clear by Remark 2.1 and Theorem 4.6.

When $s-\hat{c}>1$, let $x_{0}$ be a new variable over $R$. For each $i$, let $f_{i}^{\prime}$ be a general $d-$ form in the ideal $\left(f_{i}, x_{0}\right) \subset R\left[x_{0}\right]$. Now, consider the new homomorphism $\gamma: T \rightarrow R\left[x_{0}\right]$ induced by $z_{i} \mapsto f_{i}^{\prime}$. Notice that $\mathcal{F}^{\prime}:=\left\{f_{1}^{\prime}, \ldots, f_{s}^{\prime}\right\}$ is a set of $(\hat{c}+1)$-generic forms in $R\left[x_{0}\right]$. Whence, by induction, $\boldsymbol{F}_{\bullet} \otimes_{T} R\left[x_{0}\right]$ is a graded minimal free resolution of $R\left[x_{0}\right] / I_{a}\left(\left(\mathcal{F}^{\prime}\right)^{b}\right)^{(m)}$. Meanwhile, we have the graded isomorphism

$$
R\left[x_{0}\right] /\left(I_{a}\left(\left(\mathcal{F}^{\prime}\right)^{b}\right)^{(m)}, x_{0}\right) \cong R / I_{a}\left(\mathcal{F}^{b}\right)^{(m)} .
$$

Thus, the last piece of the proof is to show that $x_{0}$ is a non-zero-divisor of $R\left[x_{0}\right] / I_{a}\left(\left(\mathcal{F}^{\prime}\right)^{b}\right)^{(m)}$.

For this, we take arbitrary $g \in R\left[x_{0}\right]$ and assume that $x_{0} g \in I_{a}\left(\left(\mathcal{F}^{\prime}\right)^{b}\right)^{(m)}$. By Theorem 4.6 and [13, Theorem 3.6], this is equivalent to saying that $x_{0} g \in I_{c, \mathcal{F}^{\prime}}^{\left(m\left(\mu_{a}^{0}+b\left(c-c_{0}\right)\right)\right)}$ for each $c$ with $c_{0} \leq c \leq s-\delta$. However, it is shown in the proof of [13, Theorem 3.3] that $x_{0}$ is a non-zero-divisor of $R\left[x_{0}\right] / I_{c, \mathcal{F}^{\prime}}^{\left(m\left(\mu_{a}^{0}+b\left(c-c_{0}\right)\right)\right)}$. Therefore, $g \in I_{c, \mathcal{F}^{\prime}}^{\left(m\left(\mu_{a}^{0}+b\left(c-c_{0}\right)\right)\right)}$ for each c. Consequently, $g \in I_{a}\left(\left(\mathcal{F}^{\prime}\right)^{b}\right)^{(m)}$. This means that $x_{0}$ is indeed a non-zero-divisor, as expected.

Theorem 4.12. With the assumptions in Proposition 4.4, we take further $\mathcal{F}_{\boldsymbol{z}}=\left\{z_{1}, \ldots, z_{s}\right\}$ in $T=\mathbb{K}\left[z_{1}, \ldots, z_{s}\right]$. Then, we have the following properties.

(a) $\operatorname{proj} \operatorname{dim}_{R}\left(R / I_{a}\left(\mathcal{F}^{b}\right)^{(m)}\right)=s-\delta$ and $\operatorname{reg}_{R}\left(R / I_{a}\left(\mathcal{F}^{b}\right)^{(m)}\right)=m d\left(\mu_{a}^{0}+b\left(s-c_{0}\right)\right)-1$ for each positive integer $m$.

(b) The resurgence $\rho\left(I_{a}\left(\mathcal{F}^{b}\right)\right)$ coincides with the $\rho\left(I_{a}\left(\mathcal{F}_{z}^{b}\right)\right)$ given in Proposition 4.8 .

(c) For each positive integer $m$, the symbolic defect $\operatorname{sdefect}\left(I_{a}\left(\mathcal{F}^{b}\right), m\right)$ coincides with the $\operatorname{sdefect}\left(I_{a}\left(\mathcal{F}_{\boldsymbol{z}}^{b}\right), m\right)$ given in Proposition 4.9 .

Proof. (a) This follows from Proposition 4.11 and Proposition 4.7. 
(b) It is clear that $\alpha\left(I_{a}\left(\mathcal{F}^{b}\right)\right)=d \alpha\left(I_{a}\left(\mathcal{F}_{z}^{b}\right)\right)$. And by the description of the minimal generating set in (21), it is clear that $\alpha\left(I_{a}\left(\mathcal{F}^{b}\right)^{(m)}\right)=d \alpha\left(I_{a}\left(\mathcal{F}_{\boldsymbol{z}}^{b}\right)^{(m)}\right)$. Therefore,

$$
\rho\left(I_{a}\left(\mathcal{F}^{b}\right)\right) \geq \frac{\alpha\left(I_{a}\left(\mathcal{F}^{b}\right)\right)}{\widehat{\alpha}\left(I_{a}\left(\mathcal{F}^{b}\right)\right)}=\frac{d \alpha\left(I_{a}\left(\mathcal{F}_{\boldsymbol{z}}^{b}\right)\right)}{d \widehat{\alpha}\left(I_{a}\left(\mathcal{F}_{\boldsymbol{z}}^{b}\right)\right)}=\rho\left(I_{a}\left(\mathcal{F}_{\boldsymbol{z}}^{b}\right)\right) .
$$

For the reverse direction, we notice that $I_{a}\left(\mathcal{F}_{\boldsymbol{z}}^{b}\right)^{(m)} \subseteq I_{a}\left(\mathcal{F}_{z}^{b}\right)^{r}$ will imply $I_{a}\left(\mathcal{F}^{b}\right)^{(m)} \subseteq$ $I_{a}\left(\mathcal{F}^{b}\right)^{r}$, by the description in (21). Consequently, $\rho\left(I_{a}\left(\mathcal{F}^{b}\right)\right) \leq \rho\left(I_{a}\left(\mathcal{F}_{\boldsymbol{z}}^{b}\right)\right)$. And this establishes the equality.

(c) Let $\varphi$ be the homomorphism from $T$ to $R$, induced by $z_{i} \mapsto f_{i}$ for each $i$. Now, take arbitrary $\boldsymbol{z}^{\boldsymbol{\lambda}} \in G\left(I_{a}\left(\mathcal{F}_{\boldsymbol{z}}^{b}\right)^{(m)}\right)$. Without loss of generality, we may assume that $\boldsymbol{\lambda}$ is an increasing sequence and hence $\boldsymbol{\lambda} \in \Lambda\left(I_{a}\left(\mathcal{F}_{\boldsymbol{z}}^{b}\right)^{(m)}\right)$. If $\boldsymbol{z}^{\boldsymbol{\lambda}} \in I_{a}\left(\mathcal{F}_{\boldsymbol{z}}^{b}\right)^{m}$, then it is clear that $\varphi\left(\boldsymbol{z}^{\boldsymbol{\lambda}}\right) \in I_{a}\left(\mathcal{F}^{b}\right)^{m}$. On the other hand, if $\boldsymbol{z}^{\boldsymbol{\lambda}} \notin I_{a}\left(\mathcal{F}_{\boldsymbol{z}}^{b}\right)^{m}$, then by the proof of Proposition 4.9, we have

$$
|\boldsymbol{\lambda}| \leq m\left(\mu_{a}^{0}+b\left(s-\delta-c_{0}\right)\right)+\delta(m b-1)=m a-\delta<m a .
$$

Thus $\operatorname{deg}\left(\varphi\left(\boldsymbol{z}^{\boldsymbol{\lambda}}\right)\right)=d|\boldsymbol{\lambda}|<d m a=\alpha\left(I_{a}\left(\mathcal{F}^{b}\right)^{m}\right)$, implying $\varphi\left(\boldsymbol{z}^{\boldsymbol{\lambda}}\right) \notin I_{a}\left(\mathcal{F}^{b}\right)^{m}$.

Now, $I_{a}\left(\mathcal{F}^{b}\right)^{(m)} / I_{a}\left(\mathcal{F}^{b}\right)^{m}$ is generated by the set

$$
\left\{\varphi\left(\boldsymbol{z}^{\boldsymbol{\lambda}}\right): \boldsymbol{z}^{\boldsymbol{\lambda}} \in G\left(I_{a}\left(\mathcal{F}_{\boldsymbol{z}}^{b}\right)^{(m)}\right) \text { and } \boldsymbol{z}^{\boldsymbol{\lambda}} \notin I_{a}\left(\mathcal{F}_{\boldsymbol{z}}^{b}\right)^{m}\right\}
$$

by Proposition 4.11. The minimality of this generating set comes from Proposition 4.11 together with the degree reasons stated above.

Question 4.13. By [3, Corollary 5.6], we now have a closed formula for the Betti tables of the uniform $a$-fold product ideal $I_{a}\left(z_{1}^{b} \cdots z_{s}^{b}\right)$, although it is understandably complicated. What can be said regarding the general case for $I=I_{a}\left(z_{1}^{m_{1}} \cdots z_{s}^{m_{s}}\right)$ ?

\section{5. *-SYMBOLIC POWERS OF GENERAL UNIFORM $a$-FOLD PRODUCT IDEAL}

We have seen in Proposition 4.3 that the graded maximal ideal $\mathfrak{m}$ of $R=\mathbb{K}\left[x_{0}, \ldots, x_{n}\right]$ is an associate prime of $I=I_{a}\left(\mathcal{F}^{b}\right)$ when $a \leq(b-1) s+1$. Whence, $I^{(m)}=I^{m}$ for all positive integer $m$. Of course, this is not very interesting for considering containment problem of symbolic powers.

On the other hand, there is another approach in the literature for treating symbolic objects, namely, one only takes intersections over minimal primes of $I$ in (4); see for instance [25, Definition 4.3.22]. To avoid confusion in notation, we will call it the $m$-th *-symbolic power of $I$, namely, we will have

$$
I^{(m)_{*}}:=\bigcap_{\mathfrak{p} \in \operatorname{Min}(I)}\left(I^{m} R_{\mathfrak{p}} \cap R\right) .
$$

Obviously, this notion coincides with the standard one when $I$ has no embedded associate prime. And this is the case for the defining ideal of star configurations of hypersurfaces.

Now, it is time to deal with the containment problem of $*$-symbolic powers of ideal $I_{a}\left(\mathcal{F}^{b}\right)$ for $b \geq 2$ under Setting 1.1 and Setting 2.8 .

Since the forms in $\mathcal{F}$ are generic and $I_{a}\left(\mathcal{F}^{b}\right)$ has positive dimension, it follows from Theorem 2.4 that ht $\left(I_{a}\left(\mathcal{F}^{b}\right)\right)=c_{0}$ and a prime ideal $\mathfrak{p}$ is a minimal prime of $I_{a}\left(\mathcal{F}^{b}\right)$ if and only if it is a minimal prime of some complete intersection ideal $\left\langle f_{i_{1}}, \ldots, f_{i_{c_{0}}}\right\rangle$ for a unique sequence $1 \leq i_{1}<\cdots<i_{c_{0}} \leq s$. Furthermore, for this pair of prime ideal and $i$-sequence, one can verify directly that

$$
\left(I_{a}\left(\mathcal{F}^{b}\right)\right)^{m} R_{\mathfrak{p}}=\left\langle f_{i_{1}}, \ldots, f_{i_{c_{0}}}\right\rangle^{m \mu_{a}^{0}} R_{\mathfrak{p}}
$$


Since $\left\langle f_{i_{1}}, \ldots, f_{i_{0}}\right\rangle$ is a complete intersection, its power is Cohen-Macaulay and

$$
\operatorname{Ass}\left(R /\left\langle f_{i_{1}}, \ldots, f_{i_{c_{0}}}\right\rangle\right)=\operatorname{Ass}\left(R /\left\langle f_{i_{1}}, \ldots, f_{i_{c_{0}}}\right\rangle^{m \mu_{a}^{0}}\right) .
$$

This implies that

$$
\begin{aligned}
I_{a}\left(\mathcal{F}^{b}\right)^{(m)_{*}} & =\bigcap_{1 \leq i_{1}<\cdots<i_{c_{0}} \leq s}\left(\bigcap_{\mathfrak{p} \in \operatorname{Ass}\left(R /\left\langle f_{\left.\left.i_{1}, \ldots, f_{i_{0}}\right\rangle\right)}\right.\right.}\left\langle f_{i_{1}}, \ldots, f_{i_{c_{0}}}\right\rangle^{m \mu_{a}^{0}} R_{\mathfrak{p}} \cap R\right) \\
& =\bigcap_{1 \leq i_{1}<\cdots<i_{c_{0}} \leq s}\left(\bigcap_{\mathfrak{p} \in \operatorname{Ass}\left(R /\left\langle f_{i_{1}}, \ldots, f_{i_{c_{0}}}\right\rangle^{\left.m \mu_{a}^{0}\right)}\right.}\left\langle f_{i_{1}}, \ldots, f_{i_{c_{0}}}\right\rangle^{m \mu_{a}^{0}} R_{\mathfrak{p}} \cap R\right) \\
& =\bigcap_{1 \leq i_{1}<\cdots<i_{c_{0}} \leq s}^{\left\langle f_{i_{1}}, \ldots, f_{i_{c_{0}}}\right\rangle^{m \mu_{a}^{0}}} \\
& =I_{c_{0}, \mathcal{F}}^{\left(m \mu_{a}^{0}\right)} .
\end{aligned}
$$

Recall that if $I$ is a nonzero graded ideal in $R$, then $\alpha(I)$ the least degree of nonzero forms in $I$. We will in addition consider the $*$-Waldschmidt constant

$$
\widehat{\alpha}_{*}(I):=\lim _{m \rightarrow \infty} \frac{\alpha\left(I^{(m)_{*}}\right)}{m},
$$

the $*$-resurgence

$$
\rho_{*}(I)=\sup \left\{\frac{m}{r} \mid I^{(m)_{*}} \nsubseteq I^{r}\right\},
$$

and the $m$-th $*$-symbolic defect

$$
\operatorname{sdefect}_{*}(I, m)=\mu\left(I^{(m)_{*}} / I^{m}\right) .
$$

Based on the previous work of [5], [12, [17] and [18, we are able to talk about the above concepts with respect to the uniform $a$-fold product ideal $I_{a}\left(\mathcal{F}^{b}\right)$ in the following.

5.1. *-Waldschmidt constant and *-resurgence. This subsection is devoted to the study of the $*$-resurgence of $I_{a}\left(\mathcal{F}^{b}\right)$.

Remark 5.1. Notice that the containment $I \subseteq I^{(1)_{*}}$ may be strict. Thus, it is possible that $\alpha(I) \neq \alpha\left(I^{(1)_{*}}\right)$. Nevertheless, we still have some familiar properties regarding the asymptotic quantities above. We collect some pertinent preliminary facts about them here. The proofs of these facts are virtually the same as those in [2, Lemma 8.2.2] and [5], hence will be omitted here.

(a) The limit in (23) exists and $\widehat{\alpha}_{*}(I) \geq 1$.

(b) If $r \alpha(I)>\alpha\left(I^{(m)_{*}}\right)$, then $I^{r}$ does not contain $I^{(m)_{*}}$.

(c) If $m / r<\alpha(I) / \widehat{\alpha}_{*}(I)$, then for all $t \gg 0, I^{r t}$ does not contain $I^{(m t)}$. In particular, $\alpha(I) / \widehat{\alpha}_{*}(I) \leq \rho_{*}(I)$.

(d) If $I^{(m)_{*}} \subseteq I^{r}$, then $r \leq m$.

Remark 5.2. We state additionally some useful facts regarding $I_{a}\left(\mathcal{F}^{b}\right)$ for $\mathcal{F}=\left\{f_{1}, \ldots, f_{s}\right\}$ in $R=\mathbb{K}\left[x_{0}, \ldots, x_{n}\right]$. For that purpose, take $\mathcal{F}_{\boldsymbol{z}}=\left\{z_{1}, \ldots, z_{s}\right\}$ in $T=\mathbb{K}\left[z_{1}, \ldots, z_{s}\right]$. Notice that $I_{c_{0}, \mathcal{F}}^{\left(m \mu_{a}^{0}\right)}$ is the specialization of $I_{c_{0}, \mathcal{F}_{\boldsymbol{z}}}^{\left(m \mu_{a}^{0}\right)}$. 
If the forms in $\mathcal{F}$ share a common degree $d$, then, via the description in (22), we will have

$$
\alpha\left(I_{a}\left(\mathcal{F}^{b}\right)^{(m)_{*}}\right)=d \alpha\left(I_{a}\left(\mathcal{F}_{\boldsymbol{z}}^{b}\right)^{(m)_{*}}\right)=d \alpha\left(I_{c_{0}, \mathcal{F}_{\boldsymbol{z}}}^{\left(m \mu_{0}^{0}\right)}\right) .
$$

Whence,

$$
\begin{aligned}
\widehat{\alpha}_{*}\left(I_{a}\left(\mathcal{F}^{b}\right)\right) & =d \widehat{\alpha}_{*}\left(I_{a}\left(\mathcal{F}_{\boldsymbol{z}}^{b}\right)\right)=d \lim _{m \rightarrow \infty} \frac{\alpha\left(I_{a}\left(\mathcal{F}_{\boldsymbol{z}}^{b}\right)^{(m)_{*}}\right)}{m} \\
& =d \mu_{a}^{0} \lim _{m \rightarrow \infty} \frac{\alpha\left(I_{c_{0}, \mathcal{F}_{\boldsymbol{z}}}^{\left(m \mu_{0}^{0}\right)}\right)}{m \mu_{a}^{0}}=d \mu_{a}^{0} \widehat{\alpha}\left(I_{c_{0}, \mathcal{F}_{\boldsymbol{z}}}\right)=\frac{d \mu_{a}^{0} s}{c_{0}} .
\end{aligned}
$$

The last equality above uses the fact that $\widehat{\alpha}\left(I_{c_{0}, \mathcal{F}_{z}}\right)=s / c_{0}$ by [4, Theorem 7.5].

Similarly, the containment $I_{a}\left(\mathcal{F}_{\boldsymbol{z}}^{b}\right)^{(m)_{*}} \subseteq I_{a}\left(\mathcal{F}_{\boldsymbol{z}}^{b}\right)^{r}$ implies the containment $I_{a}\left(\mathcal{F}^{b}\right)^{(m)_{*}} \subseteq$ $I_{a}\left(\mathcal{F}^{b}\right)^{r}$. Consequently,

$$
\rho_{*}\left(I_{a}\left(\mathcal{F}^{b}\right)\right) \leq \rho_{*}\left(I_{a}\left(\mathcal{F}_{\boldsymbol{z}}^{b}\right)\right) .
$$

It is then natural to ask for the explicit value of $\rho_{*}\left(I_{a}\left(\mathcal{F}_{\boldsymbol{z}}^{b}\right)\right)$.

Proposition 5.3. With the assumptions in Setting[2.8, we consider the set $\mathcal{F}_{\boldsymbol{z}}=\left\{z_{1}, \ldots, z_{s}\right\}$ in $T=\mathbb{K}\left[z_{1}, \ldots, z_{s}\right]$. Then, for the uniform a-fold product ideal $I=I_{a}\left(\mathcal{F}_{\boldsymbol{z}}^{b}\right)$, we have

$$
\rho_{*}(I)=\frac{\alpha(I)}{\widehat{\alpha}_{*}(I)}=\frac{a c_{0}}{\mu_{a}^{0} s} .
$$

Proof. It follows from item (c) in Remark 5.1 and equation (24) that

$$
\rho_{*}(I) \geq \alpha(I) / \widehat{\alpha}_{*}(I)=\frac{a c_{0}}{\mu_{a}^{0} s} .
$$

It remains to prove that $\rho_{*}(I)$ is bounded above by the expected value.

In the following, we first explore relations between $m$ and $r$ such that $I^{(m)_{*}} \subseteq I^{r}$, i.e., $I_{c_{0}, \mathcal{F}_{z}}^{\left(m \mu_{a}^{0}\right)} \subseteq I_{a r}\left(\mathcal{F}_{\boldsymbol{z}}^{b r}\right)$. For this purpose, notice that these two ideals are symmetric. And partitions $\boldsymbol{\lambda} \in \mathcal{P}\left(I_{c_{0}, \mathcal{F}_{\boldsymbol{z}}}^{\left(m \mu_{a}^{0}\right)}\right)$ are characterized by the requirement $\left|\boldsymbol{\lambda}_{\leq c_{0}}\right| \geq m \mu_{a}^{0}$. Meanwhile, $\boldsymbol{\lambda} \in \mathcal{P}\left(I_{a}\left(\mathcal{F}_{\boldsymbol{z}}^{b}\right)^{r}\right)$ if and only if $\sum_{i} \min \left(\lambda_{i}, b r\right) \geq a r$.

Now, take arbitrary partition $\boldsymbol{\lambda} \in \mathcal{P}\left(I_{c_{0}, \mathcal{F}_{\boldsymbol{z}}}^{\left(m \mu_{0}^{0}\right)}\right)$. Furthermore, we may write $m \mu_{a}^{0}=q_{0} c_{0}+q_{1}$ with $1 \leq q_{1} \leq c_{0}$. Then, $\lambda_{c_{0}} \geq q_{0}+1=\left\lceil\frac{m \mu_{a}^{0}}{c_{0}}\right\rceil$. For the containment $I_{a}\left(\mathcal{F}_{\boldsymbol{z}}^{b}\right)^{(m)} \subseteq I_{a}\left(\mathcal{F}_{\boldsymbol{z}}^{b}\right)^{r}$ with $m \geq r \geq 1$, we have two cases.

(a) Suppose that $q_{0}+1<b r$.

(i) Suppose further that $q_{0}+1 \leq \lambda_{c_{0}} \leq b r$. Whence, the minimum of $\sum_{i=1}^{s} \min \left(\lambda_{i}, b r\right)$ in this case is simply $m \mu_{a}^{0}+\left(s-c_{0}\right)\left(q_{0}+1\right)$, achieved at $\boldsymbol{\lambda}=\left(q_{0}^{c_{0}-q_{1}},\left(q_{0}+\right.\right.$ $\left.1)^{s-c_{0}+q_{1}}\right)$. Thus, the requirement for the containment is

$$
m \mu_{a}^{0}+\left(s-c_{0}\right)\left(q_{0}+1\right) \geq a r,
$$

which is equivalent to asking for

$$
r \leq \frac{s\left(q_{0}+1\right)+\left(q_{1}-c_{0}\right)}{a} .
$$

(ii) Suppose instead that $q_{0}+1<b r \leq \lambda_{c_{0}}$, then the minimum of $\sum_{i=1}^{s} \min \left(\lambda_{i}, b r\right)$ in this case is $\left(s-c_{0}+1\right) b r$. Now, the requirement for the containment is

$$
\left(s-c_{0}+1\right) b r \geq a r,
$$


which holds automatically. Thus, there is no requirement in this subcase.

(b) Suppose that $b r \leq q_{0}+1$. Similar to the discussion in (ii) above, the requirement is void in this case.

To sum up, the above arguments implies that $I^{(m)_{*}} \nsubseteq I^{r}$ precisely when

$$
\left\lceil\frac{m \mu_{a}^{0}}{c_{0}}\right\rceil<b r \quad \text { and } \quad r>\frac{s\left(q_{0}+1\right)+\left(q_{1}-c_{0}\right)}{a} .
$$

Notice that for any fixed $m$, we always have $I^{(m)_{*}} \nsubseteq I^{r}$ for sufficiently large $r$. This means that the conditions above are not empty. Whence,

$$
\frac{m}{r}<\frac{a\left(\left(q_{0}+1\right) c_{0}+\left(q_{1}-c_{0}\right)\right)}{\mu_{a}^{0}\left(\left(q_{0}+1\right) s+\left(q_{1}-c_{0}\right)\right)} \leq \frac{a c_{0}}{\mu_{a}^{0} s}
$$

as $1 \leq q_{1} \leq c_{0}$ and $c_{0}<s$. In particular,

$$
\rho_{*}(I) \leq \frac{a c_{0}}{\mu_{a}^{0} s} .
$$

Combining the inequality (26), we obtain the desired formula for $\rho_{*}(I)$.

Now, we are ready for the first main result of this section.

Theorem 5.4. With the assumptions in Settings 1.1 and [2.8, we assume further that the forms in $\mathcal{F}=\left\{f_{1}, \ldots, f_{s}\right\}$ share a common degree $d$ in $\mathbb{K}\left[x_{0}, \ldots, x_{n}\right]$. Then

$$
\rho_{*}(I)=\frac{\alpha(I)}{\widehat{\alpha}_{*}(I)}=\frac{a c_{0}}{\mu_{a}^{0} s}
$$

for the ideal $I=I_{a}\left(\mathcal{F}^{b}\right)$.

Proof. Take in addition $\mathcal{F}_{z}=\left\{z_{1}, \ldots, z_{s}\right\}$ in $T=\mathbb{K}\left[z_{1}, \ldots, z_{s}\right]$. Now, it suffices to notice that

$$
\frac{a c_{0}}{\mu_{a}^{0} s}=\frac{\alpha\left(I_{a}\left(\mathcal{F}^{b}\right)\right)}{\widehat{\alpha}_{*}\left(I_{a}\left(\mathcal{F}^{b}\right)\right)} \leq \rho_{*}\left(I_{a}\left(\mathcal{F}^{b}\right)\right) \leq \rho_{*}\left(I_{a}\left(\mathcal{F}_{z}^{b}\right)\right)=\frac{a c_{0}}{\mu_{a}^{0} s},
$$

by combining the equations (24), (25) and Proposition 5.3.

5.2. *-symbolic defect. This subsection is devoted to the study of the $*$-symbolic defect of $I_{a}\left(\mathcal{F}^{b}\right)$. We start with the following observation.

Lemma 5.5 ([18, Proposition 4.10(2)]). With the assumptions in Settings 1.1, we further take $\mathcal{F}_{\boldsymbol{z}}=\left\{z_{1}, \ldots, z_{s}\right\}$ in $T=\mathbb{K}\left[z_{1}, \ldots, z_{s}\right]$. Let $\varphi: T \rightarrow R$ be the homomorphism induced by $z_{i} \mapsto f_{i}$ for each $i$. Then for each positive integer $m$, we have

$$
\left\{\varphi(g): g \in G\left(I_{c, \mathcal{F}_{z}}^{(m)}\right)\right\} \cap I_{c, \mathcal{F}}^{m}=\left\{\varphi(h)^{m}: h \in G\left(I_{c, \mathcal{F}_{z}}\right)\right\} .
$$

In particular, we have $I_{c, \mathcal{F}}^{m} \nsubseteq\left\langle f_{1}, \ldots, f_{s}\right\rangle I_{c, \mathcal{F}}^{(m)}$.

Here is the key observation for the last strike.

Proposition 5.6. With the assumptions in Settings 1.1] and 2.8, we assume further that the forms in $\mathcal{F}=\left\{f_{1}, \ldots, f_{s}\right\}$ share a common degreed in $\mathbb{K}\left[x_{0}, \ldots, x_{n}\right]$. Then the following statements are equivalent:

(a) $I_{a}\left(\mathcal{F}^{b}\right)^{m} \nsubseteq\left\langle f_{1}, \ldots, f_{s}\right\rangle I_{a}\left(\mathcal{F}^{b}\right)^{(m)_{*}}$ for all positive integer $m$;

(b) $I_{a}\left(\mathcal{F}^{b}\right)^{m} \nsubseteq\left\langle f_{1}, \ldots, f_{s}\right\rangle I_{a}\left(\mathcal{F}^{b}\right)^{(m) *}$ for some positive integer $m$;

(c) the parameter $a$ is a multiple of $b$. 
Proof. Firstly, we show that (b) $\Rightarrow(\mathrm{c})$. Let $\varphi: T \rightarrow R$ be the homomorphism induced by $z_{i} \mapsto f_{i}$ for each $i$. Since $I_{a}\left(\mathcal{F}^{b}\right)^{m}=\varphi\left(I_{a}\left(\mathcal{F}_{\boldsymbol{z}}^{b}\right)^{m}\right)$ while

$$
\left\langle f_{1}, \ldots, f_{s}\right\rangle I_{a}\left(\mathcal{F}^{b}\right)^{(m)_{*}}=\varphi\left(\left\langle z_{1}, \ldots, z_{s}\right\rangle\right) \varphi\left(I_{a}\left(\mathcal{F}_{\boldsymbol{z}}^{b}\right)^{(m)_{*}}\right)=\varphi\left(\left\langle z_{1}, \ldots, z_{s}\right\rangle I_{a}\left(\mathcal{F}_{\boldsymbol{z}}^{b}\right)^{(m)_{*}}\right),
$$

we will have $I_{a}\left(\mathcal{F}_{\boldsymbol{z}}^{b}\right)^{m} \nsubseteq\left\langle z_{1}, \ldots, z_{s}\right\rangle I_{a}\left(\mathcal{F}_{\boldsymbol{z}}^{b}\right)^{(m)_{*}}$. But it is clear that $I_{a}\left(\mathcal{F}_{\boldsymbol{z}}^{b}\right)^{m} \subseteq I_{a}\left(\mathcal{F}_{\boldsymbol{z}}^{b}\right)^{(m)_{*}}$. Now, $I_{a}\left(\mathcal{F}_{\boldsymbol{z}}^{b}\right)^{m} \nsubseteq\left\langle z_{1}, \ldots, z_{s}\right\rangle I_{a}\left(\mathcal{F}_{\boldsymbol{z}}^{b}\right)^{(m)_{*}}$ if and only if the intersection of minimal monomial generating sets $G\left(I_{a}\left(\mathcal{F}_{\boldsymbol{z}}^{b}\right)^{m}\right) \cap G\left(I_{a}\left(\mathcal{F}_{\boldsymbol{z}}^{b}\right)^{(m)_{*}}\right) \neq \varnothing$. Take any monomial $\boldsymbol{z}^{\boldsymbol{\lambda}}$ in that intersection. Without loss of generality, we may assume that $\lambda_{1} \leq \cdots \leq \lambda_{s}$. Now $\boldsymbol{\lambda} \in \Lambda\left(I_{a}\left(\mathcal{F}_{\boldsymbol{z}}^{b}\right)^{m}\right)$ and $|\boldsymbol{\lambda}|=a m$. On the other hand, since $I_{a}\left(\mathcal{F}_{\boldsymbol{z}}^{b}\right)^{(m)_{*}}=I_{c_{0}, \mathcal{F}_{\boldsymbol{z}}}^{\left(m \mu_{\boldsymbol{z}}^{0}\right)}, \boldsymbol{\lambda} \in \Lambda\left(I_{c_{0}, \mathcal{F}_{\boldsymbol{z}}}^{\left(m \mu_{a}^{0}\right)}\right)$. Consequently, $\left|\boldsymbol{\lambda}_{\leq c_{0}}\right|=m \mu_{a}^{0}$ and $\lambda_{c_{0}}=\cdots=\lambda_{s}$. Now, $|\boldsymbol{\lambda}|=a m=m \mu_{a}^{0}+\left(s-c_{0}\right) \lambda_{c_{0}}$. As $c_{0}<s$ and $a-\mu_{a}^{0}=b\left(s-c_{0}\right)$, this forces $m b=\lambda_{c_{0}}$.

Notice that $\lambda_{c_{0}} \leq\left|\boldsymbol{\lambda}_{\leq c_{0}}\right|=m \mu_{a}^{0}$ while we always have $\mu_{a}^{0} \leq b$. Therefore, the nonemptiness of the intersection forces $b=\mu_{a}^{0}$, i.e., $a$ is a multiple of $b$. And this shows the implication $(\mathrm{b}) \Rightarrow(\mathrm{c})$.

When $a=k b$ is a multiple of $b$, then $\mu_{a}^{0}=b$, and $c_{0}=s-k+1$. Now,

$$
I_{a}\left(\mathcal{F}^{b}\right)=I_{b k}\left(\mathcal{F}^{b}\right)=I_{k}(\mathcal{F})^{b}=I_{s-c_{0}+1}(\mathcal{F})^{b}=I_{c_{0}, \mathcal{F}}^{b}
$$

by (7) and (9). Consequently, $I_{a}\left(\mathcal{F}^{b}\right)^{m}=I_{c_{0}, \mathcal{F}}^{m b}$. Meanwhile, $I_{a}\left(\mathcal{F}^{b}\right)^{(m)_{*}}=I_{c_{0}, \mathcal{F}}^{(m b)}$ by (22) . Thus, Lemma 5.5 implies that $I_{a}\left(\mathcal{F}^{b}\right)^{m} \nsubseteq\left\langle f_{1}, \ldots, f_{s}\right\rangle I_{a}\left(\mathcal{F}^{b}\right)^{(m)_{*}}$. And this shows the implication $(\mathrm{c}) \Rightarrow(\mathrm{a})$,

The implication $(\mathrm{a}) \Rightarrow(\mathrm{b})$ is trivial.

Here comes the final result of this paper.

Theorem 5.7. With the assumptions in Settings 1.1] and [2.8, we assume further that $m$ is a positive integer and the forms in $\mathcal{F}=\left\{f_{1}, \ldots, f_{s}\right\}$ share a common degree $d$ in $R=\mathbb{K}\left[x_{0}, \ldots, x_{n}\right]$.

(a) If the parameter a is not a multiple of $b$, then $\operatorname{sdefect}_{*}\left(I_{a}\left(\mathcal{F}^{b}\right), m\right)$ is given by

$$
\sum_{B=\left\{b_{1}<\cdots<b_{h}\right\} \subseteq[c]}\left|\mathbb{S}_{B}\right|\left(\begin{array}{c}
s \\
c_{0}-b_{h}
\end{array}\right)\left(\begin{array}{c}
s-c_{0}+b_{h} \\
b_{h}-b_{h-1}
\end{array}\right)\left(\begin{array}{c}
s-c_{0}+b_{h-1} \\
b_{h-1}-b_{h-2}
\end{array}\right) \cdots\left(\begin{array}{c}
s-c_{0}+b_{2} \\
b_{2}-b_{1}
\end{array}\right),
$$

where $\mathbb{S}_{B}$ is the set of all distinct positive solutions to the Diophantine equation $b_{1} x_{1}+$ $\cdots+b_{h} x_{h}=m \mu_{a}^{0}$.

(b) If $a=k b$ is a multiple of $b$, then $I_{a}\left(\mathcal{F}^{b}\right)=I_{s-c_{0}+1}(\mathcal{F})^{b}$. Whence, $\operatorname{sdefect}_{*}\left(I_{a}\left(\mathcal{F}^{b}\right), m\right)$ is given by

$\sum_{B=\left\{b_{1}<\cdots<b_{h}\right\} \subseteq[c]}\left|\mathbb{S}_{B}\right|\left(\begin{array}{c}s \\ c_{0}-b_{h}\end{array}\right)\left(\begin{array}{c}s-c_{0}+b_{h} \\ b_{h}-b_{h-1}\end{array}\right)\left(\begin{array}{c}s-c_{0}+b_{h-1} \\ b_{h-1}-b_{h-2}\end{array}\right) \cdots\left(\begin{array}{c}s-c_{0}+b_{2} \\ b_{2}-b_{1}\end{array}\right)-\left(\begin{array}{c}s \\ c_{0}-1\end{array}\right)$, where $\mathbb{S}_{B}$ is defined as above with $\mu_{a}^{0}=b$.

Proof. If $a$ is not a multiple of $b$, then $I_{a}\left(\mathcal{F}^{b}\right)^{m} \subseteq\left\langle f_{1}, \ldots, f_{s}\right\rangle I_{a}\left(\mathcal{F}^{b}\right)^{(m)_{*}}$ by Proposition 5.6, Therefore, sdefect ${ }_{*}\left(I_{a}\left(\mathcal{F}^{b}\right), m\right)$ is simply the minimal number of generators of $I_{a}\left(\mathcal{F}^{b}\right)^{(m)_{*}}=$ $I_{c_{0}, \mathcal{F}}^{\left(m \mu_{a}^{0}\right)}$ which is given in [18, Corollary 4.12(1)].

Similarly, if $a=k b$ is a multiple of $b$, then we have already seen that $I_{a}\left(\mathcal{F}^{b}\right)=I_{c_{0}, \mathcal{F}}^{b}$. Since

$$
\operatorname{Min}\left(I_{a}\left(\mathcal{F}^{b}\right)\right)=\operatorname{Min}\left(I_{c_{0}, \mathcal{F}}^{b}\right)=\operatorname{Min}\left(I_{c_{0}, \mathcal{F}}\right)=\operatorname{Ass}\left(R / I_{c_{0}, \mathcal{F}}\right),
$$


this implies that $\operatorname{sdefect}_{*}\left(I_{a}\left(\mathcal{F}^{b}\right), m\right)=\operatorname{sdefect}\left(I_{c_{0}, \mathcal{F}}, m b\right)$ and the latter is given in [18, Corollary 4.12(2)].

Remark 5.8. When $s-c_{0}+1$ or $m$ is small, a more explicit description of the $*$-symbolic defect is available in [18, Section 4.2] via the information above.

Acknowledgment. The authors want to express sincere thanks to Tohăneanu and Xie for helpful discussions. The second author is partially supported by the "Anhui Initiative in Quantum Information Technologies" (No. AHY150200) and the "Fundamental Research Funds for the Central Universities".

\section{REFERENCES}

[1] B. Anzis, M. Garrousian, and Ştefan O. Tohăneanu, Generalized star configurations and the Tutte polynomial., J. Algebr. Comb. 46 (2017), 165-187.

[2] T. Bauer, S. Di Rocco, B. Harbourne, M. Kapustka, A. Knutsen, W. Syzdek, and T. Szemberg, A primer on Seshadri constants, Interactions of classical and numerical algebraic geometry, 2009, pp. 33-70.

[3] J. Biermann, H. De Alba, F. Galetto, S. Murai, U. Nagel, A. O'Keefe, T. Römer, and A. Seceleanu, Betti numbers of symmetric shifted ideals (2019), available at arXiv:1907.04288.

[4] C. Bocci, S. Cooper, E. Guardo, B. Harbourne, M. Janssen, U. Nagel, A. Seceleanu, A. Van Tuyl, and $\mathrm{T}$. Vu, The Waldschmidt constant for squarefree monomial ideals, J. Algebraic Combin. 44 (2016), 875-904.

[5] C. Bocci and B. Harbourne, Comparing powers and symbolic powers of ideals, J. Algebraic Geom. 19 (2010), 399-417.

[6] W. Bruns and J. Herzog, Cohen-Macaulay rings, Rev. ed., Cambridge Studies in Advanced Mathematics, vol. 39, Cambridge University Press, Cambridge, 1998.

[7] A. Conca and M. C. Tsakiris, Resolution of ideals associated to subspace arrangements (2019), available at arXiv: 1910.01955

[8] H. Dao, A. De Stefani, E. Grifo, C. Huneke, and L. Núñez Betancourt, Symbolic powers of ideals, Singularities and foliations. geometry, topology and applications, 2018, pp. 387-432.

[9] D. Eisenbud and M. Hochster, A Nullstellensatz with nilpotents and Zariski's main lemma on holomorphic functions, J. Algebra 58 (1979), 157-161.

[10] F. Galetto, A. V. Geramita, Y.-S. Shin, and A. Van Tuyl, The symbolic defect of an ideal, J. Pure Appl. Algebra 223 (2019), 2709-2731.

[11] M. Garrousian, A. Simis, and Ş. O. Tohăneanu, A blowup algebra for hyperplane arrangements, Algebra Number Theory 12 (2018), 1401-1429.

[12] A. V. Geramita, B. Harbourne, and J. Migliore, Star configurations in $\mathbb{P}^{n}$, J. Algebra 376 (2013), 279-299.

[13] A. V. Geramita, B. Harbourne, J. Migliore, and U. Nagel, Matroid configurations and symbolic powers of their ideals, Trans. Amer. Math. Soc. 369 (2017), 7049-7066.

[14] D. R. Grayson and M. E. Stillman, Macaulay2, a software system for research in algebraic geometry, 2018. Available at http://www.math.uiuc.edu/Macaulay2/.

[15] H. T. Hà, H. D. Nguyen, N. V. Trung, and T. N. Trung, Symbolic powers of sums of ideals, accepted to Mathematische Zeitschrift (2019).

[16] J. Herzog and T. Hibi, Monomial ideals, Graduate Texts in Mathematics, vol. 260, Springer-Verlag London Ltd., London, 2011.

[17] M. Lampa-Baczyńska and G. Malara, On the containment hierarchy for simplicial ideals, J. Pure Appl. Algebra 219 (2015), 5402-5412.

[18] P. Mantero, The structure and free resolution of the symbolic powers of star configurations of hypersurfaces (2019), available at arXiv:1907.08172.

[19] H. Matsumura, Commutative ring theory, Cambridge Studies in Advanced Mathematics, vol. 8, Cambridge University Press, Cambridge, 1986.

[20] H. D. Nguyen and N. V. Trung, Depth functions of symbolic powers of homogeneous ideals, Invent. Math. 218 (2019), 779-827. 
[21] H. Schenck and Ş. O. Tohăneanu, The Orlik-Terao algebra and 2-formality, Math. Res. Lett. 16 (2009), 171-182.

[22] Ş. O. Tohăneanu, On the de Boer-Pellikaan method for computing minimum distance, J. Symbolic Comput. 45 (2010), 965-974.

[23] - A commutative algebraic approach to the fitting problem, Proc. Amer. Math. Soc. 142 (2014), 659-666.

[24] Ş. O. Tohăneanu and Y. Xie, On the Geramita-Harbourne-Migliore conjecture (2019), available at arXiv:1906.08346.

[25] R. H. Villarreal, Monomial algebras, Second, Monographs and Research Notes in Mathematics, CRC Press, Boca Raton, FL, 2015.

The Penn State University, Department of Mathematics, Greater Allegheny Campus, McKeesport, PA, 15132, USA

E-mail address: kul20@psu.edu

Key Laboratory of Wu Wen-Tsun Mathematics, Chinese Academy of Sciences, School of Mathematical Sciences, University of Science and Technology of China, Hefei, Anhui, 230026, P.R. CHINA

E-mail address: yhshen@ustc.edu.cn 